\title{
Organocatalytic Asymmetric Synthesis of Dihydroisoquinolinones via a One-Pot Aza-Henry-Hemiaminalization-Oxidation Sequence
}

\author{
Robert Hahn \\ Ehsan Jafari \\ Gerhard Raabe \\ Dieter Enders* \\ Institute of Organic Chemistry, RWTH Aachen University, \\ Landoltweg 1, 52074 Aachen, Germany \\ enders@rwth-aachen.de
}
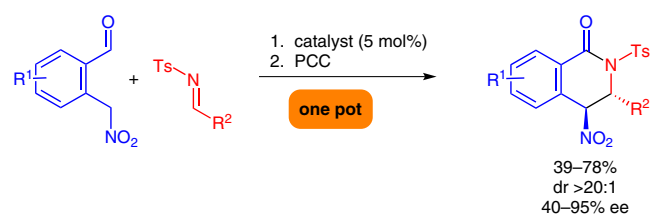

Received: 13.10 .2014

Accepted: 14.10.2014

Published online: 14.11 .2014

DOI: 10.1055/s-0034-1379398; Art ID: ss-2014-z0625-op

License terms: (c) $(1)$

Abstract The asymmetric organocatalytic one-pot synthesis of trans3,4-disubstituted 3,4-dihydroisoquinolin-1(2H)-ones is described. Starting from 2-(nitromethyl)benzaldehydes and various $\mathrm{N}$-protected aldimines, $5 \mathrm{~mol} \%$ of a quinine-based squaramide organocatalyst was used to synthesize the title compounds as virtually single diastereomers via an aza-Henry-hemiaminalization-oxidation sequence. Moderate to good yields (39-78\%) and moderate to very good enantioselectivities (40-95\% ee) were reached.

Key words organocatalysis, domino reaction, one-pot reaction, dihydroisoquinolinones, hydrogen bonding

The structural motif of the dihydroisoquinolinones is common in natural products, ${ }^{1}$ for example in thalflavine $(\mathbf{1}),^{2}(+)$-pancratistatin $(\mathbf{2})^{3}$ and (+)-plicamine (3). ${ }^{4}$ Such compounds exhibit valuable bioactivities, such as cancer cell growth inhibition ${ }^{3 c, j}$ and antiviral activity ${ }^{3 \mathrm{k}}$ for pancratistatin (2), as well as anti-inflammatory and antidepressant activity. ${ }^{\text {b }}$ Other synthetically derived dihydroisoquinolinones are the steroidomimetic drug $4,{ }^{5}$ which possesses bioactivity against certain cancer cell lines, and $\mathrm{H}_{3}$ receptor antagonist $5,{ }^{6}$ which plays a crucial role for the release of neurotransmitters and in the treatment of neuropathic pain and schizophrenia (Figure 1). Therefore, efforts have been made for the asymmetric construction of these heterocycles. ${ }^{7}$ Our group has contributed a chiral-auxiliarybased enantioselective procedure utilizing lithiated $\boldsymbol{o}$-toluamides and aldehyde SAMP or RAMP hydrazones via a 1,2addition-ring-closure sequence. ${ }^{8}$

Domino reactions are a versatile tool in organic chemistry nowadays. ${ }^{9}$ This type of reaction class allows the construction of complex molecules with a highly functionalized framework and multiple adjacent stereocen-<smiles>COc1cc2c(c3c1OCO3)CCN(C)C2=O</smiles>

thalflavine (1)<smiles>C[C@@]12c3cc4c(c(O)c3C(=O)N[C@H]1[C@@H](O)[C@H](O)[C@@H](O)[C@@H]2O)OCO4</smiles>

(+)-pancratistatin (2)<smiles></smiles>

(+)-plicamine (3)<smiles>COc1cc2c(cc1O)CCN(C(=O)c1cc(OC)c(OC)c(OC)c1)C2=O</smiles>

steroidomimetic drug<smiles>CNC(=O)c1ccc(-c2ccc3c(c2)CCN(CCN2CCCC2)C3=O)cc1</smiles>

$\mathrm{H}_{3}$ receptor antagonist

Figure 1 Selected examples of naturally and synthetically derived dihydroisoquinolinones 
ters. As a one-pot protocol, it lowers cost, reduces the amount of time required and yields more product due to reduced purification steps.

Recently, we reported the enantioselective conjugate addition of 2-(nitromethyl)benzaldehydes 6 to various nitroolefins 7 leading to functionalized 1,2,3,4-tetrahydronaphthalen-1-ols 8 via an organocatalytic nitroalkaneMichael-Henry domino reaction (Scheme 1 , a). ${ }^{10}$ We now report the first organocatalytic asymmetric synthesis of disubstituted 3,4-dihydroisoquinolin-1(2H)-ones 11 by again employing the concept of hydrogen-bonding organocatalysis. ${ }^{11}$ In the first step of the protocol, an aza-Henry ${ }^{12}$ addition of $\mathbf{6}$ to aldimines $\mathbf{9}$ is used, generating two adjacent stereocenters with the same configuration as in the previous work. A subsequent hemiaminalization ${ }^{13}$ occurs to generate the envisaged 1,2,3,4-tetrahydroisoquinolin-1-ols 10 (Scheme 1, b).

Attempts to protect the hydroxy function of the relatively sensitive hemiaminals $\mathbf{1 0}$ failed; however, an oxidation with pyridinium chlorochromate in the same pot led to the anticipated 3,4-dihydroisoquinolin-1(2H)-ones 11. With this protocol in hand, we were then able to investigate the optimal conditions for this domino transformation.

Firstly, we searched for an appropriate organocatalyst which can coordinate and activate 2-(nitromethyl)benzaldehyde (6a) and the $N$-tosyl-protected aldimine 9a to merge them to the corresponding 1,2,3,4-tetrahydroisoquinolin-1-ol 10a, followed by the oxidation with pyridinium chlorochromate. This sequence was conducted as a two-step procedure. Almost every organocatalyst we tested furnished the desired dihydroisoquinolinone 11a (Table 1). A pseudonorephedrine-derived catalyst $\mathbf{A}^{14}$ was applied to this sequence and the product was obtained in low enantioselectivity (28\% ee; Table 1, entry 1). Takemoto's catalyst B resulted in moderate $36 \%$ ee (Table 1 , entry 2 ) and the squaramide derivative $\mathbf{C}$ gave a slightly better result with
$42 \%$ ee (Table 1 , entry 3 ), suggesting that we should stick to the more reactive squaramides. After three days, no product was detected with catalyst $\mathbf{D}$, indicating that a basic amino group is needed for the deprotonation of the nitroalkane (Table 1, entry 4).

The quinine-based squaramide $\mathbf{E}$ catalyzed the domino reaction with good enantioselectivity of $65 \%$ ee (Table 1 , entry 5), whereas the related catalyst $\mathbf{F}$ with its pseudoenantiomeric structure gave only a low ee value (Table 1, entry 6). Next, we evaluated the best solvent and found that in benzene the enantioselectivity was slightly lower than in toluene (63\% ee; Table 1 , entry 7 ). The use of $m$-xylene and mesitylene as further aromatic nonpolar solvents gave lower enantioselectivities (59\% and $28 \%$ ee) with moderate yields (37\% and 43\%; Table 1, entries 8 and 9). Acetonitrile and diethyl ether were used as more polar solvents but the previous results could not be matched (11\% yield, $3 \%$ ee for $\mathrm{MeCN}$ and $40 \%$ yield, $56 \%$ ee for $\mathrm{Et}_{2} \mathrm{O}$; Table 1, entries 10 and 11). After four days, $n$-hexane gave no satisfying result and the product was obtained in $22 \%$ yield and $19 \%$ ee (Table 1 , entry 12). Switching to chlorinated solvents was also not successful, due to low yield and enantioselectivity (Table 1, entries 13-15).

Continuing the optimization of the protocol, we then checked for the best $\mathrm{N}$-protection group for the aldimines (Table 2). The $o$-nosyl and $p$-nosyl groups were chosen because of their electron-withdrawing properties, leading to more reactive aldimines (Table 2, entries 1 and 2), but both protecting groups gave lower enantioselectivities (52\% and $54 \%$ ee). With the benzyloxycarbonyl protecting group the product was obtained as a racemic mixture (Table 2, entry 3 ). Other attempts with the $N$-(tert-butoxycarbonyl)imine, the tosyl hydrazone or a benzyl protecting group gave no product at all (Table 2, entries 4-6). With the $N$-tosyl-protected imine as best substrate we tested for the optimal catalyst loading. Reducing the catalyst amount to $5 \mathrm{~mol} \%$ or

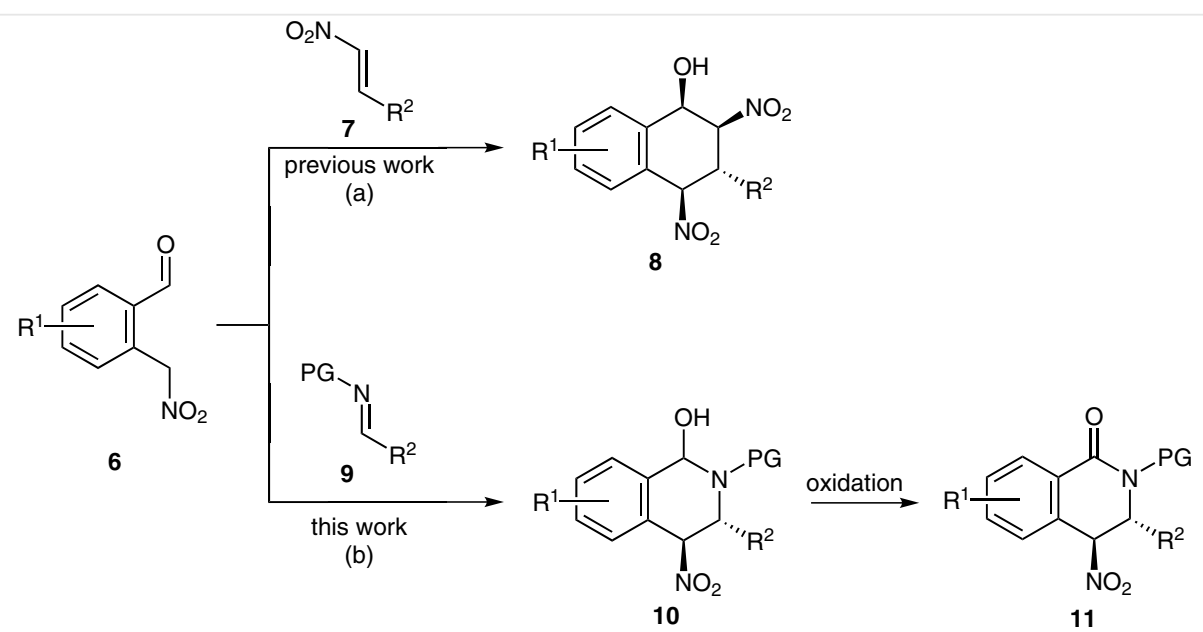

Scheme 1 2-(Nitromethyl)benzaldehydes as bifunctional substrates in asymmetric organocatalytic domino reactions 
Table 1 Catalyst and Solvent Screening for the Aza-Henry-Hemiaminalization-Oxidation Sequence To Form 3,4-Dihydroisoquinolin-1(2H)-one 11a

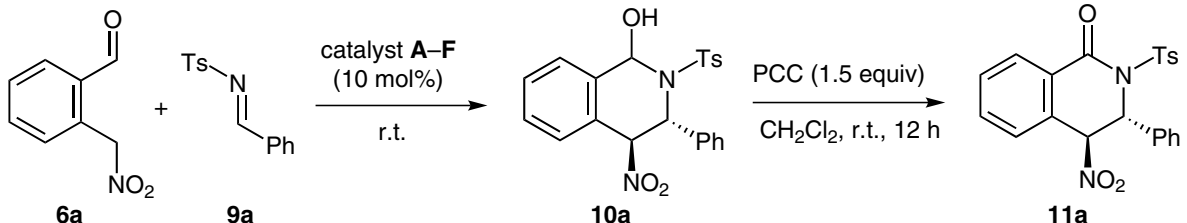<smiles>CC(c1ccccc1)N1CCCCC1</smiles><smiles>C=CC1CN2CCC1C2[C@H](Nc1c(NCC#N)c(=O)c1=O)c1ccnc2ccc(OC)cc12</smiles>

E<smiles>CN(C)[C@H]1CCCC[C@H]1NC(=S)N[Ga]</smiles>

B<smiles></smiles><smiles>FC(F)(F)c1cc(I)cc(C(F)(F)F)c1</smiles>

\begin{tabular}{|c|c|c|c|c|c|}
\hline Entry ${ }^{a}$ & Catalyst & Solvent & Time (d) & Yield ${ }^{\mathrm{b}}(\%)$ & $\mathrm{ee}^{\mathrm{c}}(\%)$ \\
\hline 1 & A & toluene & 3 & n.d. ${ }^{d}$ & 28 \\
\hline 2 & B & toluene & 3 & n.d. & $36^{e}$ \\
\hline 3 & C & toluene & 3 & n.d. & $42^{\mathrm{e}}$ \\
\hline 4 & D & toluene & 3 & 0 & - \\
\hline 5 & E & toluene & 4 & n.d. & 65 \\
\hline 6 & $\mathbf{F}$ & toluene & 3 & n.d. & $11^{\mathrm{e}}$ \\
\hline 7 & E & benzene & 3 & n.d. & 63 \\
\hline 8 & $E$ & m-xylene & 1 & 37 & 59 \\
\hline 9 & E & mesitylene & 1 & 43 & 28 \\
\hline 10 & $\mathbf{E}$ & $\mathrm{MeCN}$ & 1 & 11 & 3 \\
\hline 11 & 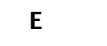 & $\mathrm{Et}_{2} \mathrm{O}$ & 1 & 40 & 56 \\
\hline 12 & E & $n$-hexane & 4 & 22 & 19 \\
\hline 13 & E & $\mathrm{CH}_{2} \mathrm{Cl}_{2}$ & 1 & 18 & 53 \\
\hline 14 & E & $\mathrm{CHCl}_{3}$ & 1 & 15 & 37 \\
\hline 15 & $\mathbf{E}$ & $\mathrm{DCE}$ & 1 & 13 & 44 \\
\hline
\end{tabular}

All reactions were performed on a 0.2 -mmol scale.

${ }^{\mathrm{b}} \mathrm{A}$ virtually pure trans-diastereomer was obtained $(\mathrm{dr}>20: 1)$; yield of isolated product after the two-step sequence.

Determined by HPLC analysis on a chiral stationary phase.

${ }^{\mathrm{d}} \mathrm{n} . \mathrm{d} .=$ not determined.

e The opposite enantiomer was obtained in excess. 
increasing to $15 \mathrm{~mol} \%$ gave similar results in yield (39\% and $41 \%$ ) and enantioselectivity (63\% and $65 \%$ ee; Table 2, entries 7 and 8). Staying with the $5 \mathrm{~mol} \%$ catalyst loading, we then screened for the best reaction temperature. Decreasing the temperature to $0{ }^{\circ} \mathrm{C}$ and $-20^{\circ} \mathrm{C}$ resulted in better yields and enantioselectivities (Table 2, entries 9 and 10). With the optimized conditions in hand (Table 2, entry 10), we then investigated the scope of this domino sequence.

The reaction of various 2-(nitromethyl)benzaldehydes $\mathbf{6}$ and $\mathrm{N}$-tosyl-protected aldimines 9 with $5 \mathrm{~mol} \%$ of the squaramide organocatalyst $\mathbf{E}$ was conducted in toluene at $-20{ }^{\circ} \mathrm{C}$. To improve the previous protocol, we then oxidized the intermediate hemiaminals directly in the same pot to afford the 3,4-dihydroisoquinolin-1(2H)-ones 11a-l as solids, which can be easily recrystallized from benzene or isopropyl alcohol (Table 3). This one-pot protocol afforded the products in modest to good yields (39-78\%) and with moderate to very good enantiomeric excesses (40-95\% ee). The model compound was finally obtained in $65 \%$ yield and $63 \%$ ee, with $95 \%$ ee after recrystallization (Table 3, 11a). Various substituents on the aromatic part of the $\mathrm{N}$-tosyl-protected aldimines $\mathbf{9}$ were tolerated, as well as on the aromatic core of the benzaldehydes $\mathbf{6}$. Alkyl or alkoxy substituents on aldimine 9 gave moderate results (Table $3, \mathbf{1 1 b}-\mathbf{d}$ ), except for the enantiomeric excess of 11d ( $94 \%$ ee).

In the case of isomers of the naphthyl-substituted aldimines, the 2-naphthyl moiety gave the better results (Table 3 , 11e and 11f). Further derivatization of the aldimine $\mathbf{9}$ with electron-withdrawing groups gave good results in each case (Table $3, \mathbf{1 1} \mathbf{g}$-i). The halogenated aromatic core of the benzaldehydes $\mathbf{6}$ yielded the corresponding products $11 \mathbf{j}$ and $11 \mathbf{k}$ in the same range as the aldimines with similar substituents on the $\mathrm{R}^{2}$ unit (Table $3, \mathbf{1 1 h}$ and $\mathbf{1 1 i}$ ); only the enantiomeric excess of product $\mathbf{1 1} \mathbf{k}$ was lower. Changing the phenyl group for an indolyl moiety in $\mathbf{9}$ led to no product. Only the racemic product could be obtained using triethylamine (Table 3, 111).

The absolute configuration of 3,4-dihydroisoquinolin$1(2 \mathrm{H})$-one 11a was determined as $(3 R, 4 S)$ by single crystal $\mathrm{X}$-ray structure analysis (Figure 2 ) ${ }^{15}$ The strongly distorted lactam unit results in a dihedral angle of $64^{\circ}$, which explains the coupling constants of $\sim 2 \mathrm{~Hz}$ of the vicinal transproton NMR signals.

Table 2 Screening for the Optimized Conditions Including Protection Group, Catalyst Loading and Temperature for the Aza-Henry-Hemiaminalization-Oxidation Sequence<smiles>O=Cc1ccccc1C[N+](=O)[O-]</smiles>

$6 a$<smiles>O=CN=Cc1ccccc1</smiles>

9

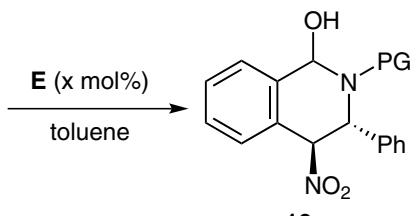

10<smiles>O=C(c1ccccc1)N1C(=O)c2ccccc2C([N+](=O)[O-])[C@H]1c1ccccc1</smiles>

11

\begin{tabular}{lllllll}
\hline Entry & PG & mol\% & Temp $\left({ }^{\circ} \mathrm{C}\right)$ & Time (d) & Yield $^{\mathrm{b}}(\%)$ & $\mathrm{ee}^{\mathrm{c}}(\%)$ \\
\hline 1 & o-nosyl & 10 & r.t. & 4 & n.d. $^{\mathrm{d}}$ & $52^{\mathrm{e}}$ \\
2 & p-nosyl & 10 & r.t. & 3 & n.d. & $54^{\mathrm{e}}$ \\
3 & Cbz & 10 & r.t. & 4 & n.d. & 0 \\
4 & Boc & 10 & r.t. & 2 & 0 & - \\
5 & NHTs & 10 & r.t. & 3 & 0 & - \\
6 & Bn & 10 & r.t. & 2 & 0 & - \\
7 & Ts & 5 & r.t. & 1 & 39 & 63 \\
8 & Ts & 15 & r.t. & 1 & 41 & 65 \\
9 & Ts & 5 & 0 & 3 & 53 & 64 \\
10 & Ts & 5 & -20 & 3 & 59 & 65 \\
\hline
\end{tabular}

${ }^{a}$ All reactions were performed on a 0.2 -mmol scale.

${ }^{b}$ A virtually pure trans-diastereomer was obtained $(\mathrm{dr}>20: 1)$; yield of isolated product after the two-step sequence.

' Determined by HPLC analysis on a chiral stationary phase, unless otherwise indicated.

${ }^{\mathrm{d}}$ n.d. $=$ not determined.

e Determined by SFC analysis on a chiral stationary phase. 
Table 3 Substrate Scope of the Aza-Henry-Hemiaminalization-Oxidation Sequence To Form the 3,4-Dihydroisoquinolin-1(2H)-ones 11a-I

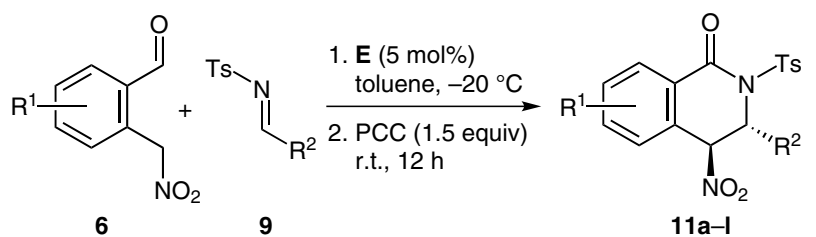

\begin{tabular}{|c|c|c|c|c|c|}
\hline $11^{\mathrm{a}}$ & $\mathrm{R}^{1}$ & $R^{2}$ & $\operatorname{Time}^{\mathrm{b}}(\mathrm{d})$ & Yieldc (\%) & $\mathrm{ee}^{\mathrm{d}}(\%)$ \\
\hline $\mathbf{a}$ & $\mathrm{H}$ & $\mathrm{Ph}$ & $3+0.5$ & 65 & $63(95)^{\mathrm{e}}$ \\
\hline b & $\mathrm{H}$ & 4-Tol & $5+0.5$ & 46 & $40^{\mathrm{e}}$ \\
\hline c & $\mathrm{H}$ & $3,4,5-(\mathrm{MeO})_{3} \mathrm{C}_{6} \mathrm{H}_{2}$ & $3.5+0.5$ & 53 & 57 \\
\hline d & $\mathrm{H}$ & $3,4-\left(\mathrm{OCH}_{2} \mathrm{O}\right) \mathrm{C}_{6} \mathrm{H}_{3}$ & $6+0.5$ & 40 & 94 \\
\hline e & $\mathrm{H}$ & 1-Naph & $3+0.5$ & 39 & 62 \\
\hline$f$ & $\mathrm{H}$ & 2-Naph & $5+0.5$ & 57 & $69(89)$ \\
\hline g & $\mathrm{H}$ & $3-\mathrm{O}_{2} \mathrm{NC}_{6} \mathrm{H}_{4}$ & $5+0.5$ & 77 & 84 \\
\hline h & $\mathrm{H}$ & $2-\mathrm{BrC}_{6} \mathrm{H}_{4}$ & $5+0.5$ & 78 & 77 \\
\hline $\mathbf{i}$ & $\mathrm{H}$ & 4- $-\mathrm{FC}_{6} \mathrm{H}_{4}$ & $3+0.5$ & 54 & 89 \\
\hline j & $6-\mathrm{Br}^{\mathrm{f}}$ & $\mathrm{Ph}$ & $5+0.5$ & 77 & 77 \\
\hline k & $7-\mathrm{F}^{\mathrm{f}}$ & $\mathrm{Ph}$ & $5+0.5$ & 52 & $60(72)$ \\
\hline$\rho^{g}$ & $\mathrm{H}$ & $N$-tosylindol-3-yl & $2+0.5$ & 44 & - \\
\hline
\end{tabular}

${ }^{a}$ All reactions were performed on a $0.5-\mathrm{mmol}$ scale.

b Reaction time for the domino plus oxidation step.

c A virtually pure trans-diastereomer was obtained ( $\mathrm{dr}>20: 1$ ); yield of isolated product after domino reaction and oxidation in one pot.

${ }^{d}$ Determined by SFC analysis on a chiral stationary phase, unless otherwise indicated; value in brackets after one recrystallization.

e Determined by HPLC analysis on a chiral stationary phase; value in brackets after one recrystallization.

${ }^{\mathrm{f}}$ Numbering refers to the product.

${ }^{g}$ Reaction with $\mathrm{Et}_{3} \mathrm{~N}$.

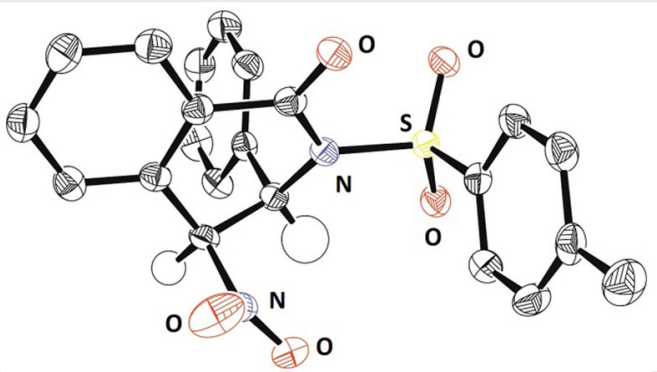

Figure 2 Determination of the absolute configuration of compound 11 a by $X$-ray crystal structure analysis ${ }^{15}$

In conclusion, we have developed the first organocatalytic asymmetric synthesis of functionalized 3,4-dihydroisoquinolin-1(2H)-ones via an aza-Henry/hemiaminalization/oxidation sequence by employing $5 \mathrm{~mol} \%$ of a quinine-based squaramide organocatalyst. This one-pot protocol utilizes easy accessible 2-(nitromethyl)benzaldehydes and $\mathrm{N}$-tosyl-protected aldimines as substrates to furnish the trans-configured title compounds with two adjacent stereocenters. The substituent pattern could be varied on the aromatic parts $\left(R^{1}, R^{2}\right)$ with electron-donating and electron-withdrawing groups, such as alkyl, alkoxy, nitro or halogen atoms. The dihydroisoquinolinones were obtained in modest to good yields (39-78\%), as virtually single diastereomers ( $d r>20: 1)$, and with moderate to very good enantiomeric excesses (40-95\% ee).

Flash column chromatography was performed on SIL G-25 UV254 (particle size $0.040-0.063 \mathrm{~mm}$, Macherey-Nagel). TLC was performed on silica gel 60 F254 plates (Merck, Darmstadt). Visualization of the developed TLC plates was performed with UV radiation $(254 \mathrm{~nm})$ or by staining with a $\mathrm{KMnO}_{4}$ solution. Elemental analyses were carried out with a Vario EL elemental analyzer. Melting points were determined with a Büchi Melting Point B-540 apparatus. Optical rotation values were measured on a Perkin-Elmer P241 polarimeter. The ee values were determined by analytical HPLC with a Hewlett-Packard 1100 Series instrument or by analytical SFC with a Thar Waters Method Station 2, using chiral stationary phases. IR spectra were recorded on a Perkin-Elmer FT-IR Spectrum 100 spectrometer. ${ }^{1} \mathrm{H}$ and ${ }^{13} \mathrm{C}$ NMR spectra were measured at ambient temperature with Varian Innova 400 or Varian Innova 600 spectrometers using TMS as internal standard. Mass spectra were recorded on a Finnigan SSQ7000 (EI $70 \mathrm{eV}$ ) spectrometer, high-resolution mass spectra on a Finnigan MAT 95 spectrometer and high-resolution ESI mass spectra on a Thermo Fisher Scientific LTQ Orbitrap XL spectrometer.

\section{Asymmetric Synthesis of 3,4-Dihydroisoquinolin-1(2H)-one De- rivatives 11a-l; General Procedure}

In a glass vial equipped with a magnetic stirrer bar, a 2-(nitromethyl)benzaldehyde 6 ( $0.5 \mathrm{mmol}, 1.0$ equiv), an $N$-tosyl-protected aldimine 9 ( $0.55 \mathrm{mmol}, 1.1$ equiv) and organocatalyst $\mathbf{E}$ ( $5 \mathrm{~mol} \%$ ) were dissolved in toluene $(1 \mathrm{~mL})$. After the mixture was stirred at $-20{ }^{\circ} \mathrm{C}$ for the appropriate time, PCC $(0.75 \mathrm{mmol})$ was added. This mixture was stirred at r.t. and monitored by TLC. After completion of the reaction, the solvent was evaporated and the crude mixture was directly purified on silica gel ( $n$-hexane-EtOAc, 5:1 to 2:1) to afford the product $\mathbf{1 1}$ as a colorless solid.

\section{(3R,4S)-4-Nitro-3-phenyl-2-tosyl-3,4-dihydroisoquinolin-1(2H)- one (11a)}

Synthesized according to the general procedure from 2-(nitromethyl)benzaldehyde (6a; $82 \mathrm{mg}, 0.50 \mathrm{mmol})$ and $N$-tosylimine 9a (140 $\mathrm{mg}, 0.54 \mathrm{mmol})$. The oxidation step was conducted with PCC (160 mg, $0.74 \mathrm{mmol}$ ); yield: $136 \mathrm{mg}$ (65\%); colorless solid; mp $142{ }^{\circ} \mathrm{C}$ (benzene).

$[\alpha]_{\mathrm{D}}^{20}+4.5\left(\right.$ c $\left.1.01, \mathrm{CHCl}_{3}\right) ; 95 \%$ ee; $R_{f}=0.66$ ( $n$-hexane-EtOAc, $\left.1: 1\right)$.

IR (film): 3060, 2982, 2921, 2315, 2066, 1687, 1597, 1554, 1495, 1454, 1354, 1246, 1165, 1117, 1066, 1011, 907, 845, 802, 761, 728, $700 \mathrm{~cm}^{-1}$.

${ }^{1} \mathrm{H}$ NMR $\left(600 \mathrm{MHz}, \mathrm{CDCl}_{3}\right): \delta=2.40\left(\mathrm{~s}, 3 \mathrm{H}, \mathrm{CH}_{3}\right), 5.73\left(\mathrm{~d},{ }^{3} \mathrm{~J}=1.8 \mathrm{~Hz}, 1\right.$ $\left.\mathrm{H}, \mathrm{CHNO}_{2}\right), 6.81\left(\mathrm{~d},{ }^{3} \mathrm{~J}=1.8 \mathrm{~Hz}, 1 \mathrm{H}, \mathrm{CHPh}\right), 7.19-7.21(\mathrm{~m}, 2 \mathrm{H}$, $\left.2 \times \mathrm{CH}_{\mathrm{Ar}}\right), 7.27-7.29\left(\mathrm{~m}, 5 \mathrm{H}, 5 \times \mathrm{CH}_{\mathrm{Ar}}\right), 7.32-7.34\left(\mathrm{~m}, 1 \mathrm{H}, \mathrm{CH}_{\mathrm{Ar}}\right), 7.55-$ $7.58\left(\mathrm{~m}, 2 \mathrm{H}, 2 \times \mathrm{CH}_{\mathrm{Ar}}\right), 7.87\left(\mathrm{~d}, 3^{3}=8.4 \mathrm{~Hz}, 2 \mathrm{H}, 2 \times \mathrm{CH}_{\mathrm{Ar}}\right), 8.17-8.18$ $\left(\mathrm{m}, 1 \mathrm{H}, \mathrm{CH}_{\mathrm{Ar}}\right)$. 
${ }^{13} \mathrm{C} \mathrm{NMR}\left(150 \mathrm{MHz}, \mathrm{CDCl}_{3}\right): \delta=21.7\left(\mathrm{CH}_{3}\right), 61.2(\mathrm{CHPh}), 86.0\left(\mathrm{CHNO}_{2}\right)$, $126.4\left(2 \times \mathrm{CH}_{\mathrm{Ar}}\right), 127.6\left(\mathrm{C}_{\mathrm{Ar}}\right), 128.8\left(\mathrm{C}_{\mathrm{Ar}}\right), 129.0\left(\mathrm{CH}_{\mathrm{Ar}}\right), 129.2\left(2 \times \mathrm{CH}_{\mathrm{Ar}}\right)$, $129.3\left(\mathrm{CH}_{\mathrm{Ar}}\right), 129.3\left(2 \times \mathrm{CH}_{\mathrm{Ar}}\right), 129.5\left(2 \times \mathrm{CH}_{\mathrm{Ar}}\right), 130.6\left(\mathrm{CH}_{\mathrm{Ar}}\right), 131.6$ $\left(\mathrm{CH}_{\mathrm{Ar}}\right), 134.3\left(\mathrm{CH}_{\mathrm{Ar}}\right), 135.1\left(\mathrm{C}_{\mathrm{Ar}}\right), 135.2\left(\mathrm{C}_{\mathrm{Ar}}\right), 145.4\left(\mathrm{C}_{\mathrm{Ar}}\right), 161.1(\mathrm{C}=0)$. MS (EI, $70 \mathrm{eV}): m / z(\%)=423(1)[\mathrm{M}+\mathrm{H}]^{+}, 376(8), 358(21), 312(100)$, 221 (14), 155 (16), 91 (34), 77 (2).

HRMS: $m / z[\mathrm{M}+\mathrm{Na}]^{+}$calcd for $\mathrm{C}_{22} \mathrm{H}_{18} \mathrm{~N}_{2} \mathrm{O}_{5} \mathrm{SNa}^{+}$: 445.0829; found: 445.0821 .

\section{(3R,4S)-3-(4-Methylphenyl)-4-nitro-2-tosyl-3,4-dihydroisoquino- lin-1(2H)-one (11b)}

Synthesized according to the general procedure from 2-(nitromethyl)benzaldehyde (6a; $83 \mathrm{mg}, 0.50 \mathrm{mmol}$ ) and $N$-tosylimine $\mathbf{9 b}(163$ $\mathrm{mg}, 0.60 \mathrm{mmol}$ ). The oxidation step was conducted with PCC (166 mg, $0.77 \mathrm{mmol})$; yield: $102 \mathrm{mg}(46 \%)$; colorless solid; $\mathrm{mp} 109{ }^{\circ} \mathrm{C}(i-\mathrm{PrOH})$. $[\alpha]_{\mathrm{D}}{ }^{20}+17.2\left(\mathrm{c} 0.95, \mathrm{CHCl}_{3}\right) ; 40 \%$ ee; $R_{f}=0.72$ (n-hexane-EtOAc, $\left.1: 1\right)$. IR (film): 2987, 2921, 2311, 2075, 1976, 1690, 1596, 1555, 1453 , 1353, 1299, 1246, 1168, 1116, 1064, 1013, 910, 806, $720 \mathrm{~cm}^{-1}$.

$\left.{ }^{1} \mathrm{H} \mathrm{NMR} \mathrm{(400} \mathrm{MHz,} \mathrm{CDCl}_{3}\right): \delta=2.26\left(\mathrm{~s}, 3 \mathrm{H}, \mathrm{CH}_{3}\right), 2.39\left(\mathrm{~s}, 3 \mathrm{H}, \mathrm{CH}_{3}\right)$, $5.69\left(\mathrm{~d},{ }^{3} \mathrm{~J}=1.9 \mathrm{~Hz}, 1 \mathrm{H}, \mathrm{CHNO}_{2}\right), 6.75\left(\mathrm{~d},{ }^{3} \mathrm{~J}=1.9 \mathrm{~Hz}, 1 \mathrm{H}, \mathrm{CHAr}\right), 7.07$ $\left(\mathrm{m}, 4 \mathrm{H}, 4 \times \mathrm{CH}_{\mathrm{Ar}}\right), 7.28\left(\mathrm{~d},{ }^{3} \mathrm{~J}=8.3 \mathrm{~Hz}, 2 \mathrm{H}, 2 \times \mathrm{CH}_{\mathrm{Ar}}\right), 7.30-7.32(\mathrm{~m}, 1 \mathrm{H}$, $\left.\mathrm{CH}_{\mathrm{Ar}}\right), 7.53-7.57\left(\mathrm{~m}, 2 \mathrm{H}, 2 \times \mathrm{CH}_{\mathrm{Ar}}\right), 7.87\left(\mathrm{~d},{ }^{3} \mathrm{~J}=8.4 \mathrm{~Hz}, 2 \mathrm{H}, 2 \times \mathrm{CH}_{\mathrm{Ar}}\right)$, 8.14-8.18 (m, $\left.1 \mathrm{H}, \mathrm{CH}_{\mathrm{Ar}}\right)$.

${ }^{13} \mathrm{C}$ NMR (100 MHz, $\left.\mathrm{CDCl}_{3}\right): \delta=21.0\left(\mathrm{CH}_{3}\right), 21.7\left(\mathrm{CH}_{3}\right), 61.0(\mathrm{CHAr})$, $86.1\left(\mathrm{CHNO}_{2}\right), 126.2\left(2 \times \mathrm{CH}_{\mathrm{Ar}}\right), 127.7\left(\mathrm{C}_{\mathrm{Ar}}\right), 128.8\left(\mathrm{C}_{\mathrm{Ar}}\right), 129.2$ $\left(2 \times \mathrm{CH}_{\mathrm{Ar}}\right), 129.2\left(\mathrm{CH}_{\mathrm{Ar}}\right), 129.5\left(2 \times \mathrm{CH}_{\mathrm{Ar}}\right), 129.9\left(2 \times \mathrm{CH}_{\mathrm{Ar}}\right), 130.5$ $\left(\mathrm{CH}_{\mathrm{Ar}}\right), 131.6\left(\mathrm{CH}_{\mathrm{Ar}}\right), 132.2\left(\mathrm{C}_{\mathrm{Ar}}\right), 134.2\left(\mathrm{CH}_{\mathrm{Ar}}\right), 135.2\left(\mathrm{C}_{\mathrm{Ar}}\right), 138.9\left(\mathrm{C}_{\mathrm{Ar}}\right)$, $145.3\left(\mathrm{C}_{\mathrm{Ar}}\right), 161.1(\mathrm{C}=\mathrm{O})$.

MS (EI, $70 \mathrm{eV}): m / z(\%)=437(1)[\mathrm{M}+\mathrm{H}]^{+}, 390(17), 372(28), 326$ (100), 235 (33), 155 (13), 91 (8).

HRMS: $m / z[\mathrm{M}+\mathrm{H}]^{+}$calcd for $\mathrm{C}_{23} \mathrm{H}_{21} \mathrm{~N}_{2} \mathrm{O}_{5} \mathrm{~S}^{+}$: 437.1166; found: 437.1163.

\section{(3R,4S)-4-Nitro-2-tosyl-3-(3,4,5-trimethoxyphenyl)-3,4-dihydro- isoquinolin-1(2H)-one (11c)}

Synthesized according to the general procedure from 2-(nitromethyl)benzaldehyde (6a; $83 \mathrm{mg}, 0.50 \mathrm{mmol}$ ) and $N$-tosylimine 9c (199 $\mathrm{mg}, 0.57 \mathrm{mmol}$ ). The oxidation step was conducted with PCC (168 mg, $0.78 \mathrm{mmol}$ ); yield: $136 \mathrm{mg}$ (53\%); colorless solid; mp $190{ }^{\circ} \mathrm{C}(i-\mathrm{PrOH})$. $[\alpha]_{\mathrm{D}}{ }^{20}-13.6$ (c 0.98, $\mathrm{CHCl}_{3}$ ); 57\% ee; $R_{f}=0.43$ ( $n$-hexane-EtOAc, $1: 1$ ). IR (film): 2259, 1693, 1596, 1557, 1463, 1358, 1303, 1249, 1167, $1118,1067,1014,908,846,806,727 \mathrm{~cm}^{-1}$.

${ }^{1} \mathrm{H}$ NMR $\left(600 \mathrm{MHz}, \mathrm{CDCl}_{3}\right): \delta=2.41\left(\mathrm{~s}, 3 \mathrm{H}, \mathrm{CH}_{3}\right), 3.68(\mathrm{~s}, 6 \mathrm{H}$, $\left.2 \times \mathrm{OCH}_{3}\right), 3.78\left(\mathrm{~s}, 3 \mathrm{H}, \mathrm{OCH}_{3}\right), 5.74\left(\mathrm{~d},{ }^{3} \mathrm{~J}=1.9 \mathrm{~Hz}, 1 \mathrm{H}, \mathrm{CHNO}_{2}\right), 6.35(\mathrm{~s}$, $\left.2 \mathrm{H}, 2 \times \mathrm{CH}_{\mathrm{Ar}}\right), 6.73\left(\mathrm{~d},{ }^{3} \mathrm{~J}=1.8 \mathrm{~Hz}, 1 \mathrm{H}, \mathrm{CHAr}\right), 7.30\left(\mathrm{~d},{ }^{3} \mathrm{~J}=8.4 \mathrm{~Hz}, 2 \mathrm{H}\right.$, $\left.2 \times \mathrm{CH}_{\mathrm{Ar}}\right), 7.37-7.38\left(\mathrm{~m}, 1 \mathrm{H}, \mathrm{CH}_{\mathrm{Ar}}\right), 7.57-7.62\left(\mathrm{~m}, 2 \mathrm{H}, 2 \times \mathrm{CH}_{\mathrm{Ar}}\right), 7.89$ $\left(\mathrm{d},{ }^{3} \mathrm{~J}=8.3 \mathrm{~Hz}, 2 \mathrm{H}, 2 \times \mathrm{CH}_{\mathrm{Ar}}\right), 8.16-8.18\left(\mathrm{~m}, 1 \mathrm{H}, \mathrm{CH}_{\mathrm{Ar}}\right)$.

${ }^{13} \mathrm{C}$ NMR $\left(150 \mathrm{MHz}, \mathrm{CDCl}_{3}\right): \delta=21.7\left(\mathrm{CH}_{3}\right), 56.0\left(2 \times \mathrm{OCH}_{3}\right), 60.8$ $\left(\mathrm{OCH}_{3}\right), 61.3(\mathrm{CHAr}), 85.9\left(\mathrm{CHNO}_{2}\right), 103.5\left(2 \times \mathrm{CH}_{\mathrm{Ar}}\right), 127.8\left(\mathrm{C}_{\mathrm{Ar}}\right), 128.7$ $\left(\mathrm{C}_{\mathrm{Ar}}\right), 129.0\left(\mathrm{CH}_{\mathrm{Ar}}\right), 129.3\left(2 \times \mathrm{CH}_{\mathrm{Ar}}\right), 129.5\left(2 \times \mathrm{CH}_{\mathrm{Ar}}\right), 130.5\left(\mathrm{C}_{\mathrm{Ar}}\right), 130.7$ $\left(\mathrm{CH}_{\mathrm{Ar}}\right), 131.7\left(\mathrm{CH}_{\mathrm{Ar}}\right), 134.4\left(\mathrm{CH}_{\mathrm{Ar}}\right), 135.1\left(\mathrm{C}_{\mathrm{Ar}}\right), 138.2\left(\mathrm{C}_{\mathrm{Ar}}\right), 145.5\left(\mathrm{C}_{\mathrm{Ar}}\right)$, $153.7\left(2 \times \mathrm{C}_{\mathrm{Ar}}\right), 161.1(\mathrm{C}=\mathrm{O})$.

MS (EI, $70 \mathrm{eV}): m / z(\%)=512(24)\left[\mathrm{M}^{+}\right], 466$ (67), 401 (2), $296(20)$, 284 (26), 311 (100), 155 (7), 91 (11).
HRMS: $m / z[\mathrm{M}+\mathrm{H}]^{+}$calcd for $\mathrm{C}_{25} \mathrm{H}_{25} \mathrm{~N}_{2} \mathrm{O}_{8} \mathrm{~S}^{+}$: 513.1326; found: 513.1326 .

(3R,4S)-3-(3,4-Methylenedioxyphenyl)-4-nitro-2-tosyl-3,4-dihydroisoquinolin-1(2H)-one (11d)

Synthesized according to the general procedure from 2-(nitromethyl)benzaldehyde (6a; $82 \mathrm{mg}, 0.50 \mathrm{mmol}$ ) and $N$-tosylimine 9d (166 $\mathrm{mg}, 0.55 \mathrm{mmol})$. The oxidation step was conducted with PCC (156 mg, $0.72 \mathrm{mmol}$ ); yield: $92 \mathrm{mg}$ (40\%); colorless solid; $\mathrm{mp} 172{ }^{\circ} \mathrm{C}(i-\mathrm{PrOH})$.

$[\alpha]_{\mathrm{D}}^{20}+6.4$ ( c 0.97, $\left.\mathrm{CHCl}_{3}\right) ; 94 \%$ ee; $R_{f}=0.63$ ( $n$-hexane-EtOAc, $\left.1: 1\right)$.

IR (film): 2984, 2906, 2285, 2068, 1982, 1690, 1598, 1556, 1495 , $1447,1354,1244,1166,1029,914,810,722 \mathrm{~cm}^{-1}$.

${ }^{1} \mathrm{H}$ NMR (400 MHz, $\left.\mathrm{CDCl}_{3}\right): \delta=2.40\left(\mathrm{~s}, 3 \mathrm{H}, \mathrm{CH}_{3}\right), 5.66\left(\mathrm{~d},{ }^{3} \mathrm{~J}=1.9 \mathrm{~Hz}, 1\right.$ $\left.\mathrm{H}, \mathrm{CHNO}_{2}\right), 5.91\left(\mathrm{~d},{ }^{2} J=1.5 \mathrm{~Hz}, 1 \mathrm{H}, \mathrm{CH}_{\mathrm{a}} \mathrm{H}_{\mathrm{b}}\right), 5.91\left(\mathrm{~d},{ }^{2} J=1.5 \mathrm{~Hz}, 1 \mathrm{H}\right.$, $\left.\mathrm{CH}_{\mathrm{a}} \mathrm{H}_{\mathrm{b}}\right), 6.62\left(\mathrm{~s}, 1 \mathrm{H}, \mathrm{CH}_{\mathrm{Ar}}\right), 6.67-6.68\left(\mathrm{~m}, 3 \mathrm{H}, \mathrm{CHAr}, 2 \times \mathrm{CH}_{\mathrm{Ar}}\right), 7.28-$ $7.30\left(\mathrm{~d},{ }^{3} \mathrm{~J}=8.2 \mathrm{~Hz}, 2 \mathrm{H}, 2 \times \mathrm{CH}_{\mathrm{Ar}}\right), 7.32-7.36\left(\mathrm{~m}, 1 \mathrm{H}, \mathrm{CH}_{\mathrm{Ar}}\right), 7.55-7.60$ $\left(\mathrm{m}, 2 \mathrm{H}, 2 \times \mathrm{CH}_{\mathrm{Ar}}\right), 7.89\left(\mathrm{~d},{ }^{3} \mathrm{~J}=8.4 \mathrm{~Hz}, 2 \mathrm{H}, 2 \times \mathrm{CH}_{\mathrm{Ar}}\right), 8.14-8.18(\mathrm{~m}, 1 \mathrm{H}$, $\left.\mathrm{CH}_{\mathrm{Ar}}\right)$.

${ }^{13} \mathrm{C} \mathrm{NMR}\left(150 \mathrm{MHz}, \mathrm{CDCl}_{3}\right): \delta=21.7\left(\mathrm{CH}_{3}\right), 60.9(\mathrm{CHAr}), 86.0\left(\mathrm{CHNO}_{2}\right)$, 101.6 $\left(\mathrm{CH}_{2}\right), 106.7\left(\mathrm{CH}_{\mathrm{Ar}}\right), 108.8\left(\mathrm{CH}_{\mathrm{Ar}}\right), 120.3\left(\mathrm{CH}_{\mathrm{Ar}}\right), 127.6\left(\mathrm{C}_{\mathrm{Ar}}\right), 128.7$ $\left(\mathrm{C}_{\mathrm{Ar}}\right), 129.0\left(\mathrm{C}_{\mathrm{Ar}}\right), 129.2\left(2 \times \mathrm{CH}_{\mathrm{Ar}}\right), 129.3\left(\mathrm{CH}_{\mathrm{Ar}}\right), 129.5\left(2 \times \mathrm{CH}_{\mathrm{Ar}}\right), 130.5$ $\left(\mathrm{CH}_{\mathrm{Ar}}\right), 131.7\left(\mathrm{CH}_{\mathrm{Ar}}\right), 134.3\left(\mathrm{CH}_{\mathrm{Ar}}\right), 135.1\left(\mathrm{C}_{\mathrm{Ar}}\right), 145.4\left(\mathrm{C}_{\mathrm{Ar}}\right), 148.1\left(\mathrm{C}_{\mathrm{Ar}}\right)$, $148.4\left(\mathrm{C}_{\mathrm{Ar}}\right), 160.9(\mathrm{C}=\mathrm{O})$.

MS (EI, $70 \mathrm{eV}): m / z(\%)=466(16)\left[\mathrm{M}^{+}\right], 420$ (100), $419(18), 356$ (8), 355 (12), 265 (87), 264 (15), 238 (27), 237 (42), 155 (11), 91 (21).

HRMS: $m / z \quad[\mathrm{M}+\mathrm{H}]^{+}$calcd for $\mathrm{C}_{23} \mathrm{H}_{19} \mathrm{~N}_{2} \mathrm{O}_{7} \mathrm{~S}^{+}$: 467.0907; found: 467.0903 .

\section{(3R,4S)-3-(1-Naphthyl)-4-nitro-2-tosyl-3,4-dihydroisoquinolin-} 1(2H)-one (11e)

Synthesized according to the general procedure from 2-(nitromethyl)benzaldehyde $(\mathbf{6 a} ; 82 \mathrm{mg}, 0.50 \mathrm{mmol})$ and $N$-tosylimine $9 \mathbf{9 e}(170$ $\mathrm{mg}, 0.55 \mathrm{mmol})$. The oxidation step was conducted with PCC (146 mg, $0.68 \mathrm{mmol}$ ); yield: $92 \mathrm{mg}$ (39\%); colorless solid; $\mathrm{mp} 188{ }^{\circ} \mathrm{C}(i-\mathrm{PrOH})$.

$[\alpha]_{\mathrm{D}}{ }^{20}-115.2\left(\mathrm{c} 0.72, \mathrm{CHCl}_{3}\right) ; 62 \%$ ee; $R_{f}=0.70$ ( $n$-hexane-EtOAc, $1: 1$ ). IR (film): 3074, 1733, 1687, 1598, 1552, 1512, 1458, 1403, 1361, 1294, 1254, 1174, 1116, 1090, 1066, 999, 903, 845, 799, 769, $714 \mathrm{~cm}^{-1}$. ${ }^{1} \mathrm{H} \mathrm{NMR}\left(600 \mathrm{MHz}, \mathrm{CDCl}_{3}\right): \delta=2.42\left(\mathrm{~s}, 3 \mathrm{H}, \mathrm{CH}_{3}\right), 5.92\left(\mathrm{~d},{ }^{3} \mathrm{~J}=1.7 \mathrm{~Hz}, 1\right.$ $\left.\mathrm{H}, \mathrm{CHNO}_{2}\right), 7.08\left(\mathrm{~d},{ }^{3} \mathrm{~J}=7.2 \mathrm{~Hz}, 1 \mathrm{H}, \mathrm{CH}_{\mathrm{Ar}}\right), 7.15\left(\mathrm{~d},{ }^{3} \mathrm{~J}=7.6 \mathrm{~Hz}, 1 \mathrm{H}\right.$, $\left.\mathrm{CH}_{\mathrm{Ar}}\right), 7.23\left(\mathrm{dd},{ }^{3} J=7.7,7.7 \mathrm{~Hz}, 1 \mathrm{H}, \mathrm{CH}_{\mathrm{Ar}}\right), 7.31\left(\mathrm{~d},{ }^{3} \mathrm{~J}=8.2 \mathrm{~Hz}, 2 \mathrm{H}\right.$, $2 \times \mathrm{CH}_{\mathrm{Ar}}$ ), 7.50 (ddd, ${ }^{3} \mathrm{~J}=7.7,7.7 \mathrm{~Hz},{ }^{4} \mathrm{~J}=1.2 \mathrm{~Hz}, 1 \mathrm{H}, \mathrm{CH}_{\mathrm{Ar}}$ ), 7.57 (ddd, $\left.{ }^{3} J=7.7,7.7 \mathrm{~Hz},{ }^{4} \mathrm{~J}=0.9 \mathrm{~Hz}, 1 \mathrm{H}, \mathrm{CH}_{\mathrm{Ar}}\right), 7.62\left(\mathrm{dd},{ }^{3} \mathrm{~J}=7.7,7.7 \mathrm{~Hz}, 1 \mathrm{H}\right.$, $\left.\mathrm{CH}_{\mathrm{Ar}}\right), 7.65(\mathrm{~s}, 1 \mathrm{H}, \mathrm{CHAr}), 7.77-7.80\left(\mathrm{~m}, 2 \mathrm{H}, 2 \times \mathrm{CH}_{\mathrm{Ar}}\right), 7.91-7.94(\mathrm{~m}, 3$ $\left.\mathrm{H}, 3 \times \mathrm{CH}_{\mathrm{Ar}}\right), 8.24\left(\mathrm{dd},{ }^{3} \mathrm{~J}=8.0 \mathrm{~Hz},{ }^{4} \mathrm{~J}=1.0 \mathrm{~Hz}, 1 \mathrm{H}, \mathrm{CH}_{\mathrm{Ar}}\right), 8.39\left(\mathrm{~d},{ }^{3} \mathrm{~J}=8.5\right.$ $\left.\mathrm{Hz}, 1 \mathrm{H}, \mathrm{CH}_{\mathrm{Ar}}\right)$.

${ }^{13} \mathrm{C} \mathrm{NMR}\left(150 \mathrm{MHz}, \mathrm{CDCl}_{3}\right): \delta=21.7\left(\mathrm{CH}_{3}\right), 58.9(\mathrm{CHAr}), 84.5\left(\mathrm{CHNO}_{2}\right)$, $121.4\left(\mathrm{CH}_{\mathrm{Ar}}\right), 125.0\left(\mathrm{CH}_{\mathrm{Ar}}\right), 125.2\left(\mathrm{CH}_{\mathrm{Ar}}\right), 126.5\left(\mathrm{CH}_{\mathrm{Ar}}\right), 127.5\left(\mathrm{C}_{\mathrm{Ar}}\right)$, $128.0\left(\mathrm{CH}_{\mathrm{Ar}}\right), 128.5\left(\mathrm{C}_{\mathrm{Ar}}\right), 129.2\left(\mathrm{C}_{\mathrm{Ar}}\right), 129.3\left(2 \times \mathrm{CH}_{\mathrm{Ar}}\right), 129.3\left(\mathrm{CH}_{\mathrm{Ar}}\right)$, $129.6\left(2 \times \mathrm{CH}_{\mathrm{Ar}}\right), 129.7\left(\mathrm{C}_{\mathrm{Ar}}\right), 129.7\left(\mathrm{CH}_{\mathrm{Ar}}\right), 129.8\left(\mathrm{CH}_{\mathrm{Ar}}\right), 130.8\left(\mathrm{CH}_{\mathrm{Ar}}\right)$, $131.6\left(\mathrm{CH}_{\mathrm{Ar}}\right), 134.1\left(\mathrm{C}_{\mathrm{Ar}}\right), 134.2\left(\mathrm{CH}_{\mathrm{Ar}}\right), 135.2\left(\mathrm{C}_{\mathrm{Ar}}\right), 145.5\left(\mathrm{C}_{\mathrm{Ar}}\right), 161.6$ $(\mathrm{C}=0)$.

MS (EI, $70 \mathrm{eV}): m / z(\%)=472(4)\left[\mathrm{M}^{+}\right], 426(83), 408$ (4), 362 (36), 361 (14), 271 (92), 270 (83), 255 (48), 254 (37), 244 (52), 243 (53), 242 (15), 241 (18), 215 (67), 155 (24), 91 (100), 65 (24).

HRMS: $m / z[\mathrm{M}+\mathrm{H}]^{+}$calcd for $\mathrm{C}_{26} \mathrm{H}_{21} \mathrm{~N}_{2} \mathrm{O}_{5} \mathrm{~S}^{+}$: 473.1166; found: 473.1163 . 
(3R,4S)-3-(2-Naphthyl)-4-nitro-2-tosyl-3,4-dihydroisoquinolin1(2H)-one (11f)

Synthesized according to the general procedure from 2-(nitromethyl)benzaldehyde (6a; $82 \mathrm{mg}, 0.50 \mathrm{mmol}$ ) and $N$-tosylimine $\mathbf{9 f}$ (177 $\mathrm{mg}, 0.57 \mathrm{mmol}$ ). The oxidation step was conducted with PCC (162 mg, $0.75 \mathrm{mmol}$ ); yield: $133 \mathrm{mg}$ (57\%); colorless solid; mp $170{ }^{\circ} \mathrm{C}(i$-PrOH $)$. $[\alpha]_{\mathrm{D}}{ }^{20}+31.6\left(c \mathrm{c} 05, \mathrm{CHCl}_{3}\right) ; 89 \%$ ee; $R_{f}=0.68$ ( $n$-hexane-EtOAc, $1: 1$ ). IR (film): 3055, 2321, 2089, 1914, 1677, 1597, 1551, 1458, 1401, 1359, 1303, 1246, 1166, 1115, 1065, 1011, 962, 907, 845, 807, 736, $706 \mathrm{~cm}^{-1}$.

${ }^{1} \mathrm{H}$ NMR $\left(600 \mathrm{MHz}, \mathrm{CDCl}_{3}\right): \delta=2.40\left(\mathrm{~s}, 3 \mathrm{H}, \mathrm{CH}_{3}\right), 5.85\left(\mathrm{~d},{ }^{3} \mathrm{~J}=1.8 \mathrm{~Hz}, 1\right.$ $\mathrm{H}, \mathrm{CHNO}_{2}$ ), 6.98 (br s, $\left.1 \mathrm{H}, \mathrm{CHAr}\right), 7.27-7.31\left(\mathrm{~m}, 4 \mathrm{H}, 4 \times \mathrm{CH}_{\mathrm{Ar}}\right), 7.45-$ $7.49\left(\mathrm{~m}, 2 \mathrm{H}, 2 \times \mathrm{CH}_{\mathrm{Ar}}\right), 7.53\left(\mathrm{dd},{ }^{3} \mathrm{~J}=1.5,7.7 \mathrm{~Hz}, 1 \mathrm{H}, \mathrm{CH}_{\mathrm{Ar}}\right), 7.53(\mathrm{dd}$, $\left.{ }^{3} J=7.7 \mathrm{~Hz},{ }^{4} J=1.1 \mathrm{~Hz}, 1 \mathrm{H}, \mathrm{CH}_{\mathrm{Ar}}\right), 7.65\left(\mathrm{~s}, 1 \mathrm{H}, \mathrm{CH}_{\mathrm{Ar}}\right), 7.69-7.71(\mathrm{~m}, 1 \mathrm{H}$, $\left.\mathrm{CH}_{\mathrm{Ar}}\right), 7.77-7.79\left(\mathrm{~m}, 2 \mathrm{H}, 2 \times \mathrm{CH}_{\mathrm{Ar}}\right), 7.91\left(\mathrm{~d},{ }^{3} \mathrm{~J}=8.3 \mathrm{~Hz}, 2 \mathrm{H}, 2 \times \mathrm{CH}_{\mathrm{Ar}}\right)$, $8.21\left(\mathrm{dd},{ }^{3} \mathrm{~J}=7.6 \mathrm{~Hz},{ }^{4} \mathrm{~J}=1.3 \mathrm{~Hz}, 1 \mathrm{H}, \mathrm{CH}_{\mathrm{Ar}}\right)$

$\left.{ }^{13} \mathrm{C} \mathrm{NMR} \mathrm{(150} \mathrm{MHz,} \mathrm{CDCl}_{3}\right): \delta=21.7\left(\mathrm{CH}_{3}\right), 61.4(\mathrm{CHAr}), 85.9\left(\mathrm{CHNO}_{2}\right)$, $123.1\left(\mathrm{CH}_{\mathrm{Ar}}\right), 126.1\left(\mathrm{CH}_{\mathrm{Ar}}\right), 126.9\left(\mathrm{CH}_{\mathrm{Ar}}\right), 127.0\left(\mathrm{CH}_{\mathrm{Ar}}\right), 127.6\left(\mathrm{C}_{\mathrm{Ar}}\right)$, 127.6 $\left(\mathrm{CH}_{\mathrm{Ar}}\right), 128.2\left(\mathrm{CH}_{\mathrm{Ar}}\right), 128.8\left(\mathrm{C}_{\mathrm{Ar}}\right), 129.3\left(2 \times \mathrm{CH}_{\mathrm{Ar}}\right), 129.3\left(\mathrm{CH}_{\mathrm{Ar}}\right)$, $129.4\left(\mathrm{CH}_{\mathrm{Ar}}\right), 129.6\left(2 \times \mathrm{CH}_{\mathrm{Ar}}\right), 130.5\left(\mathrm{CH}_{\mathrm{Ar}}\right), 131.7\left(\mathrm{CH}_{\mathrm{Ar}}\right), 132.5\left(\mathrm{C}_{\mathrm{Ar}}\right)$, $133.1\left(2 \times \mathrm{C}_{\mathrm{Ar}}\right), 134.3\left(\mathrm{CH}_{\mathrm{Ar}}\right), 135.1\left(\mathrm{C}_{\mathrm{Ar}}\right), 145.5\left(\mathrm{C}_{\mathrm{Ar}}\right), 161.2(\mathrm{C}=0)$.

MS (EI, $70 \mathrm{eV}): m / z(\%)=472(6)\left[\mathrm{M}^{+}\right], 426(100), 408$ (5), 362 (29), 361 (23), 271 (32), 244 (25), 215 (36), 155 (21), 91 (22), 65 (18).

HRMS: $m / z[\mathrm{M}+\mathrm{H}]^{+}$calcd for $\mathrm{C}_{26} \mathrm{H}_{21} \mathrm{~N}_{2} \mathrm{O}_{5} \mathrm{~S}^{+}$: 473.1166; found: 473.1161.

\section{(3R,4S)-4-Nitro-3-(3-nitrophenyl)-2-tosyl-3,4-dihydroisoquino- lin-1(2H)-one (11g)}

Synthesized according to the general procedure from 2-(nitromethyl)benzaldehyde (6a; $82 \mathrm{mg}, 0.50 \mathrm{mmol}$ ) and $\mathrm{N}$-tosylimine $\mathbf{9 g}$ (174 $\mathrm{mg}, 0.57 \mathrm{mmol}$ ). The oxidation step was conducted with PCC (156 mg, $0.72 \mathrm{mmol}$ ); yield: $180 \mathrm{mg}$ (77\%); colorless solid; mp $189^{\circ} \mathrm{C}(i-\mathrm{PrOH})$. $[\alpha]_{\mathrm{D}}^{20}-58.0$ (c 0.96, $\mathrm{CHCl}_{3}$ ); 84\% ee; $R_{f}=0.63$ ( $n$-hexane-EtOAc, $1: 1$ ). IR (film): 3309, 3088, 2920, 2850, 2096, 1736, 1679, 1593, 1529, $1351,1248,1166,1062,1011,904,839,806,730 \mathrm{~cm}^{-1}$.

${ }^{1} \mathrm{H}$ NMR $\left(600 \mathrm{MHz}, \mathrm{CDCl}_{3}\right): \delta=2.42\left(\mathrm{~s}, 3 \mathrm{H}, \mathrm{CH}_{3}\right), 5.74\left(\mathrm{~d},{ }^{3} \mathrm{~J}=2.0 \mathrm{~Hz}, 1\right.$ $\left.\mathrm{H}, \mathrm{CHNO}_{2}\right), 6.92\left(\mathrm{~d},{ }^{3} \mathrm{~J}=1.8 \mathrm{~Hz}, 1 \mathrm{H}, \mathrm{CHAr}\right), 7.33\left(\mathrm{~d},{ }^{3} \mathrm{~J}=8.1 \mathrm{~Hz}, 2 \mathrm{H}\right.$, $\left.2 \times \mathrm{CH}_{\mathrm{Ar}}\right), 7.35-7.38\left(\mathrm{~m}, 1 \mathrm{H}, \mathrm{CH}_{\mathrm{Ar}}\right), 7.51\left(\mathrm{dd},{ }^{3} \mathrm{~J}=8.0,8.0 \mathrm{~Hz}, 1 \mathrm{H}, \mathrm{CH}_{\mathrm{Ar}}\right)$, 7.58-7.63 (m, $\left.3 \mathrm{H}, 3 \times \mathrm{CH}_{\mathrm{Ar}}\right), 7.94\left(\mathrm{~d},{ }^{3} \mathrm{~J}=8.3 \mathrm{~Hz}, 2 \mathrm{H}, 2 \times \mathrm{CH}_{\mathrm{Ar}}\right), 8.13-$ $8.14\left(\mathrm{~m}, 1 \mathrm{H}, \mathrm{CH}_{\mathrm{Ar}}\right), 8.15-8.17\left(\mathrm{~m}, 1 \mathrm{H}, \mathrm{CH}_{\mathrm{Ar}}\right), 8.18-8.21\left(\mathrm{~m}, 1 \mathrm{H}, \mathrm{CH}_{\mathrm{Ar}}\right)$. ${ }^{13} \mathrm{C} \mathrm{NMR}\left(150 \mathrm{MHz}, \mathrm{CDCl}_{3}\right): \delta=21.4\left(\mathrm{CH}_{3}\right), 60.4(\mathrm{CHAr}), 85.3\left(\mathrm{CHNO}_{2}\right)$, $121.5\left(\mathrm{CH}_{\mathrm{Ar}}\right), 124.0\left(\mathrm{CH}_{\mathrm{Ar}}\right), 126.9\left(\mathrm{C}_{\mathrm{Ar}}\right), 128.5\left(\mathrm{C}_{\mathrm{Ar}}\right), 129.4\left(2 \times \mathrm{CH}_{\mathrm{Ar}}\right)$, 129.6 $\left(3 \times \mathrm{CH}_{\mathrm{Ar}}\right), 130.6\left(\mathrm{CH}_{\mathrm{Ar}}\right), 130.7\left(\mathrm{CH}_{\mathrm{Ar}}\right), 132.1\left(\mathrm{CH}_{\mathrm{Ar}}\right), 132.2\left(\mathrm{CH}_{\mathrm{Ar}}\right)$, $134.6\left(\mathrm{CH}_{\mathrm{Ar}}\right), 134.7\left(\mathrm{C}_{\mathrm{Ar}}\right), 137.7\left(\mathrm{C}_{\mathrm{Ar}}\right), 145.9\left(\mathrm{C}_{\mathrm{Ar}}\right), 148.7\left(\mathrm{C}_{\mathrm{Ar}}\right), 160.6$ $(\mathrm{C}=0)$.

MS (EI, $70 \mathrm{eV}): m / z(\%)=468(1)[\mathrm{M}+\mathrm{H}]^{+}, 421(2), 403$ (10), 357 (100), 311 (6), 155 (21), 91 (14).

HRMS: $m / z[\mathrm{M}+\mathrm{H}]^{+}$calcd for $\mathrm{C}_{22} \mathrm{H}_{18} \mathrm{~N}_{3} \mathrm{O}_{7} \mathrm{~S}^{+}$: 468.0860; found: 468.0855 .

\section{(3R,4S)-3-(2-Bromophenyl)-4-nitro-2-tosyl-3,4-dihydroisoquino- lin-1(2H)-one (11h)}

Synthesized according to the general procedure from 2-(nitromethyl)benzaldehyde (6a; $83 \mathrm{mg}, 0.50 \mathrm{mmol})$ and $N$-tosylimine $\mathbf{9 h}(182$ $\mathrm{mg}, 0.54 \mathrm{mmol})$. The oxidation step was conducted with PCC (161 mg, $0.75 \mathrm{mmol})$; yield: $197 \mathrm{mg}$ (78\%); colorless solid; $\mathrm{mp} 203{ }^{\circ} \mathrm{C}(i$-PrOH). $[\alpha]_{\mathrm{D}}^{20}-82.2$ (c 0.99, $\mathrm{CHCl}_{3}$ ); 77\% ee; $R_{f}=0.70$ ( $n$-hexane-EtOAc, $1: 1$ ).
IR (film): 2921, 2848, 2105, 1690, 1595, 1553, 1508, 1458, 1422, $1353,1243,1166,1117,1070,1013,975,907,810,711 \mathrm{~cm}^{-1}$.

${ }^{1} \mathrm{H}$ NMR $\left(600 \mathrm{MHz}, \mathrm{CDCl}_{3}\right): \delta=2.42\left(\mathrm{~s}, 3 \mathrm{H}, \mathrm{CH}_{3}\right), 5.83\left(\mathrm{~d},{ }^{3} \mathrm{~J}=1.7 \mathrm{~Hz}, 1\right.$ $\left.\mathrm{H}, \mathrm{CHNO}_{2}\right), 6.96\left(\mathrm{dd},{ }^{3} J=7.6 \mathrm{~Hz},{ }^{4} J=1.6 \mathrm{~Hz}, 1 \mathrm{H}, \mathrm{CH}_{\mathrm{Ar}}\right), 7.11\left(\mathrm{~d},{ }^{3} J=1.4\right.$ $\mathrm{Hz}, 1 \mathrm{H}, \mathrm{CHAr}), 7.13-7.18\left(\mathrm{~m}, 2 \mathrm{H}, 2 \times \mathrm{CH}_{\mathrm{Ar}}\right), 7.33\left(\mathrm{~d},{ }^{3} \mathrm{~J}=8.2 \mathrm{~Hz}, 2 \mathrm{H}\right.$, $\left.2 \times \mathrm{CH}_{\mathrm{Ar}}\right), 7.37-7.38\left(\mathrm{~m}, 1 \mathrm{H}, \mathrm{CH}_{\mathrm{Ar}}\right), 7.55-7.60\left(\mathrm{~m}, 2 \mathrm{H}, 2 \times \mathrm{CH}_{\mathrm{Ar}}\right), 7.64$ $\left(\mathrm{dd},{ }^{3} \mathrm{~J}=7.8 \mathrm{~Hz},{ }^{4} \mathrm{~J}=1.4 \mathrm{~Hz}, 1 \mathrm{H}, \mathrm{CH}_{\mathrm{Ar}}\right), 7.98\left(\mathrm{~d},{ }^{3} \mathrm{~J}=8.4 \mathrm{~Hz}, 2 \mathrm{H}\right.$, $\left.2 \times \mathrm{CH}_{\mathrm{Ar}}\right), 8.18-8.21\left(\mathrm{~m}, 1 \mathrm{H}, \mathrm{CH}_{\mathrm{Ar}}\right)$.

${ }^{13} \mathrm{C} \mathrm{NMR}\left(150 \mathrm{MHz}, \mathrm{CDCl}_{3}\right): \delta=21.7\left(\mathrm{CH}_{3}\right), 60.6(\mathrm{CHAr}), 83.3\left(\mathrm{CHNO}_{2}\right)$, $122.1\left(\mathrm{C}_{\mathrm{Ar}}\right), 127.4\left(\mathrm{C}_{\mathrm{Ar}}\right), 128.2\left(\mathrm{CH}_{\mathrm{Ar}}\right), 128.2\left(\mathrm{CH}_{\mathrm{Ar}}\right), 128.3\left(\mathrm{C}_{\mathrm{Ar}}\right), 129.2$ $\left(\mathrm{CH}_{\mathrm{Ar}}\right), 129.3\left(2 \times \mathrm{CH}_{\mathrm{Ar}}\right), 129.7\left(2 \times \mathrm{CH}_{\mathrm{Ar}}\right), 130.6\left(\mathrm{CH}_{\mathrm{Ar}}\right), 130.9\left(\mathrm{CH}_{\mathrm{Ar}}\right)$, $131.7\left(\mathrm{CH}_{\mathrm{Ar}}\right), 133.8\left(\mathrm{C}_{\mathrm{Ar}}\right), 133.9\left(\mathrm{CH}_{\mathrm{Ar}}\right), 134.4\left(\mathrm{CH}_{\mathrm{Ar}}\right), 135.1\left(\mathrm{C}_{\mathrm{Ar}}\right), 145.6$ $\left(\mathrm{C}_{\mathrm{Ar}}\right), 161.3(\mathrm{C}=0)$.

MS (EI, $70 \mathrm{eV}): m / z(\%)=456 / 454(9 / 9)\left[\mathrm{M}-\mathrm{NO}_{2}\right]^{+}, 438 / 436(13 / 13)$, 392/390 (99/100), 311 (4), 310 (6), 301/299 (10/10), 155 (22), 91 (24). HRMS: $m / z[\mathrm{M}+\mathrm{H}]^{+}$calcd for $\mathrm{C}_{22} \mathrm{H}_{18} \mathrm{~N}_{2} \mathrm{O}_{5} \mathrm{SBr}^{+}$: 501.0114; found: 501.0114 .

(3R,4S)-3-(4-Fluorophenyl)-4-nitro-2-tosyl-3,4-dihydroisoquinolin-1(2H)-one (11i)

Synthesized according to the general procedure from 2-(nitromethyl)benzaldehyde (6a; $82 \mathrm{mg}, 0.50 \mathrm{mmol}$ ) and $\mathrm{N}$-tosylimine $\mathbf{9 i}(152$ $\mathrm{mg}, 0.55 \mathrm{mmol})$. The oxidation step was conducted with PCC $(167 \mathrm{mg}$, $0.78 \mathrm{mmol}$ ); yield: $118 \mathrm{mg}$ (54\%); colorless solid; $\mathrm{mp} 188^{\circ} \mathrm{C}(i-\mathrm{PrOH})$. $[\alpha]_{\mathrm{D}}{ }^{20}+4.7\left(c 1.01, \mathrm{CHCl}_{3}\right) ; 89 \%$ ee; $R_{f}=0.70$ ( $n$-hexane-EtOAc, $1: 1$ ). IR (film): 3098, 2923, 2850, 2602, 2323, 2111, 1989, 1734, 1688, $1600,1549,1509,1459,1360,1308,1273,1232,1169,1112,1084$, $1062,1010,918,832,769,717 \mathrm{~cm}^{-1}$.

${ }^{1} \mathrm{H} \mathrm{NMR}\left(600 \mathrm{MHz}, \mathrm{CDCl}_{3}\right): \delta=2.41\left(\mathrm{~s}, 3 \mathrm{H}, \mathrm{CH}_{3}\right), 5.76\left(\mathrm{~d},{ }^{3} J=1.8 \mathrm{~Hz}, 1\right.$ $\left.\mathrm{H}, \mathrm{CHNO}_{2}\right), 6.78(\mathrm{~s}, 1 \mathrm{H}, \mathrm{CHAr}), 6.98\left(\mathrm{dd},{ }^{3} J_{\mathrm{H}, \mathrm{F}}=8.3 \mathrm{~Hz},{ }^{3} J_{\mathrm{H}, \mathrm{H}}=8.3 \mathrm{~Hz}, 2\right.$ $\left.\mathrm{H}, 2 \times \mathrm{CH}_{\mathrm{Ar}}\right), 7.19\left(\mathrm{dd},{ }^{3} J_{\mathrm{H}, \mathrm{H}}=8.4 \mathrm{~Hz},{ }^{4} \mathrm{~J}_{\mathrm{H}, \mathrm{F}}=4.8 \mathrm{~Hz}, 2 \mathrm{H}, 2 \times \mathrm{CH}_{\mathrm{Ar}}\right), 7.30$ $\left(\mathrm{d},{ }^{3} \mathrm{~J}=8.3 \mathrm{~Hz}, 2 \mathrm{H}, 2 \times \mathrm{CH}_{\mathrm{Ar}}\right), 7.33-7.36\left(\mathrm{~m}, 1 \mathrm{H}, \mathrm{CH}_{\mathrm{Ar}}\right), 7.57-7.60(\mathrm{~m}, 2$ $\left.\mathrm{H}, 2 \times \mathrm{CH}_{\mathrm{Ar}}\right), 7.88\left(\mathrm{~d},{ }^{3} \mathrm{~J}=8.2 \mathrm{~Hz}, 2 \mathrm{H}, 2 \times \mathrm{CH}_{\mathrm{Ar}}\right), 8.16-8.19(\mathrm{~m}, 1 \mathrm{H}$, $\left.\mathrm{CH}_{\mathrm{Ar}}\right)$.

${ }^{13} \mathrm{C} \mathrm{NMR}\left(150 \mathrm{MHz}, \mathrm{CDCl}_{3}\right): \delta=21.7\left(\mathrm{CH}_{3}\right), 60.6(\mathrm{CHAr}), 85.9\left(\mathrm{CHNO}_{2}\right)$, $116.4\left(\mathrm{~d},{ }^{2} J_{\mathrm{C}, \mathrm{F}}=22.2 \mathrm{~Hz}, 2 \times \mathrm{CH}_{\mathrm{Ar}}\right), 127.4\left(\mathrm{C}_{\mathrm{Ar}}\right), 128.3\left(\mathrm{~d},{ }^{3} J_{\mathrm{C}, \mathrm{F}}=8.5 \mathrm{~Hz}\right.$, $\left.2 \times \mathrm{CH}_{\mathrm{Ar}}\right), 128.7\left(\mathrm{C}_{\mathrm{Ar}}\right), 129.3\left(2 \times \mathrm{CH}_{\mathrm{Ar}}\right), 129.4\left(\mathrm{CH}_{\mathrm{Ar}}\right), 129.6\left(2 \times \mathrm{CH}_{\mathrm{Ar}}\right)$, $130.6\left(\mathrm{CH}_{\mathrm{Ar}}\right), 131.1\left(\mathrm{~d},{ }^{4} J_{\mathrm{C}, \mathrm{F}}=3.1 \mathrm{~Hz}, \mathrm{C}_{\mathrm{Ar}}\right), 131.8\left(\mathrm{CH}_{\mathrm{Ar}}\right), 134.4\left(\mathrm{CH}_{\mathrm{Ar}}\right)$, $135.0\left(\mathrm{C}_{\mathrm{Ar}}\right), 145.6\left(\mathrm{C}_{\mathrm{Ar}}\right), 160.8(\mathrm{C}=\mathrm{O}), 163.8\left(\mathrm{~d},{ }^{1} \mathrm{~J}_{\mathrm{C}, \mathrm{F}}=247.7 \mathrm{~Hz}, \mathrm{C}_{\mathrm{Ar}} \mathrm{F}\right)$.

${ }^{19} \mathrm{~F}$ NMR $\left(565 \mathrm{MHz}, \mathrm{CDCl}_{3}\right): \delta=-112.0\left(\right.$ dddd, ${ }^{3} J_{\mathrm{H}, \mathrm{F}}=8.5,8.5 \mathrm{~Hz},{ }^{4} J_{\mathrm{H}, \mathrm{F}}=$ $\left.5.0,5.0 \mathrm{~Hz}, \mathrm{C}_{\mathrm{Ar}} \mathrm{F}\right)$.

MS (EI, $70 \mathrm{eV}): m / z(\%)=394(5)\left[\mathrm{M}-\mathrm{NO}_{2}\right]^{+}, 376$ (16), 330 (100), 239 (9), 212 (17), 183 (25), 155 (13), 91 (19).

HRMS: $m / z[\mathrm{M}+\mathrm{H}]^{+}$calcd for $\mathrm{C}_{22} \mathrm{H}_{18} \mathrm{~N}_{2} \mathrm{O}_{5} \mathrm{SF}^{+}$: 441.0915; found: 441.0910.

Anal. Calcd for $\mathrm{C}_{22} \mathrm{H}_{17} \mathrm{~N}_{2} \mathrm{O}_{5} \mathrm{SF}$ : C, 59.99; H, 3.89; N, 6.36. Found: C, 59.83; $\mathrm{H}, 4.11 ; \mathrm{N}, 6.35$.

(3R,4S)-6-Bromo-4-nitro-3-phenyl-2-tosyl-3,4-dihydroisoquinolin-1(2H)-one (11j)

Synthesized according to the general procedure from 4-bromo-2-(nitromethyl)benzaldehyde (6j; $122 \mathrm{mg}, 0.50 \mathrm{mmol}$ ) and $N$-tosylimine 9a (144 mg, $0.56 \mathrm{mmol}$ ). The oxidation step was conducted with PCC (176 mg, $0.82 \mathrm{mmol}$ ); yield: $192 \mathrm{mg}(77 \%)$; colorless solid; mp $128^{\circ} \mathrm{C}$ $(i-\mathrm{PrOH})$

$[\alpha]_{\mathrm{D}}^{20}+0.5\left(c 1.03, \mathrm{CHCl}_{3}\right) ; 77 \%$ ee; $R_{f}=0.76(n$-hexane-EtOAc, $1: 1)$. 
IR (film): 3069, 2985, 2928, 2102, 1731, 1693, 1592, 1558, 1494, 1453, 1360, 1294, 1244, 1167, 1124, 1080, 1047, 911, 846, 810, 761, $731,698 \mathrm{~cm}^{-1}$

${ }^{1} \mathrm{H} \mathrm{NMR}\left(600 \mathrm{MHz}, \mathrm{CDCl}_{3}\right): \delta=2.41\left(\mathrm{~s}, 3 \mathrm{H}, \mathrm{CH}_{3}\right), 5.66\left(\mathrm{~d},{ }^{3} \mathrm{~J}=2.0 \mathrm{~Hz}, 1\right.$ $\left.\mathrm{H}, \mathrm{CHNO}_{2}\right), 6.81\left(\mathrm{~d},{ }^{3} \mathrm{~J}=2.0 \mathrm{~Hz}, 1 \mathrm{H}, \mathrm{CHPh}\right), 7.18-7.20(\mathrm{~m}, 2 \mathrm{H}$, $\left.2 \times \mathrm{CH}_{\mathrm{Ar}}\right), 7.28-7.31\left(\mathrm{~m}, 5 \mathrm{H}, 5 \times \mathrm{CH}_{\mathrm{Ar}}\right), 7.48\left(\mathrm{~d},{ }^{4} \mathrm{~J}=1.8 \mathrm{~Hz}, 1 \mathrm{H}, \mathrm{CH}_{\mathrm{Ar}}\right)$, $7.70\left(\mathrm{dd},{ }^{3} \mathrm{~J}=8.5 \mathrm{~Hz},{ }^{4} \mathrm{~J}=1.9 \mathrm{~Hz}, 1 \mathrm{H}, \mathrm{CH}_{\mathrm{Ar}}\right), 7.86\left(\mathrm{~d},{ }^{3} \mathrm{~J}=8.4 \mathrm{~Hz}, 2 \mathrm{H}\right.$, $\left.2 \times \mathrm{CH}_{\mathrm{Ar}}\right), 8.03\left(\mathrm{~d},{ }^{3} \mathrm{~J}=8.5 \mathrm{~Hz}, 1 \mathrm{H}, \mathrm{CH}_{\mathrm{Ar}}\right)$.

${ }^{13} \mathrm{C} \mathrm{NMR}\left(150 \mathrm{MHz}, \mathrm{CDCl}_{3}\right): \delta=21.7\left(\mathrm{CH}_{3}\right), 61.2(\mathrm{CHPh}), 85.4\left(\mathrm{CHNO}_{2}\right)$, $126.3\left(2 \times \mathrm{CH}_{\mathrm{Ar}}\right), 127.6\left(\mathrm{C}_{\mathrm{Ar}}\right), 129.1\left(\mathrm{C}_{\mathrm{Ar}}\right), 129.2\left(\mathrm{CH}_{\mathrm{Ar}}\right), 129.2\left(\mathrm{C}_{\mathrm{Ar}}\right)$, $129.3\left(2 \times \mathrm{CH}_{\mathrm{Ar}}\right), 129.5\left(2 \times \mathrm{CH}_{\mathrm{Ar}}\right), 129.6\left(2 \times \mathrm{CH}_{\mathrm{Ar}}\right), 130.7\left(\mathrm{CH}_{\mathrm{Ar}}\right), 133.5$ $\left(\mathrm{CH}_{\mathrm{Ar}}\right), 134.7\left(\mathrm{C}_{\mathrm{Ar}}\right), 134.9\left(\mathrm{C}_{\mathrm{Ar}}\right), 135.0\left(\mathrm{CH}_{\mathrm{Ar}}\right), 145.6\left(\mathrm{C}_{\mathrm{Ar}}\right), 160.4(\mathrm{C}=\mathrm{O})$.

MS (EI, $70 \mathrm{eV}): m / z(\%)=456 / 454(2 / 2)\left[\mathrm{M}-\mathrm{NO}_{2}\right]^{+}, 438 / 436(7 / 8)$, 392/390 (46/48), 376 (4), 312 (100), 301/299 (5/5), 221 (16), $195(20)$, 194 (15), 165 (40), 155 (57), 91 (94).

HRMS: $m / z[\mathrm{M}+\mathrm{H}]^{+}$calcd for $\mathrm{C}_{22} \mathrm{H}_{18} \mathrm{~N}_{2} \mathrm{O}_{5} \mathrm{SBr}^{+}$: 501.0114; found: 501.0113 .

\section{(3R,4S)-7-Fluoro-4-nitro-3-phenyl-2-tosyl-3,4-dihydroisoquino- lin-1(2H)-one (11k)}

Synthesized according to the general procedure from 5-fluoro-2-(nitromethyl)benzaldehyde (6k; $68 \mathrm{mg}, 0.37 \mathrm{mmol}$ ) and $\mathrm{N}$-tosylimine $\mathbf{9 a}$ (110 mg, $0.42 \mathrm{mmol}$ ). The oxidation step was conducted with PCC (146 mg, $0.68 \mathrm{mmol}$ ); yield: $85 \mathrm{mg}(52 \%)$; colorless solid; $\mathrm{mp} 190{ }^{\circ} \mathrm{C}$ $(i-\mathrm{PrOH})$

$[\alpha]_{\mathrm{D}}^{20}+4.3\left(\right.$ c $\left.0.58, \mathrm{CHCl}_{3}\right) ; 72 \%$ ee; $R_{f}=0.75$ ( $n$-hexane-EtOAc, $\left.1: 1\right)$.

IR (film): 3331, 3076, 2918, 2850, 2694, 2293, 2067, 1941, 1688, 1597, 1555, 1497, 1437, 1355, 1264, 1163, 1085, 1026, 928, 847, 807, $743 \mathrm{~cm}^{-1}$.

${ }^{1} \mathrm{H}$ NMR $\left(600 \mathrm{MHz}, \mathrm{CDCl}_{3}\right): \delta=2.41\left(\mathrm{~s}, 3 \mathrm{H}, \mathrm{CH}_{3}\right), 5.70\left(\mathrm{~d},{ }^{3} \mathrm{~J}=1.9 \mathrm{~Hz}, 1\right.$ $\left.\mathrm{H}, \mathrm{CHNO}_{2}\right), 6.81\left(\mathrm{~d},{ }^{3} \mathrm{~J}=1.9 \mathrm{~Hz}, 1 \mathrm{H}, \mathrm{CHPh}\right), 7.18-7.19(\mathrm{~m}, 2 \mathrm{H}$, $\left.2 \times \mathrm{CH}_{\mathrm{Ar}}\right), 7.24-7.27\left(\mathrm{~m}, 1 \mathrm{H}, \mathrm{CH}_{\mathrm{Ar}}\right), 7.28-7.30\left(\mathrm{~m}, 5 \mathrm{H}, 5 \times \mathrm{CH}_{\mathrm{Ar}}\right), 7.34$ $\left(\mathrm{dd},{ }^{3} J_{\mathrm{H}, \mathrm{H}}=8.4 \mathrm{~Hz},{ }^{4} \mathrm{~J}_{\mathrm{H}, \mathrm{F}}=4.8 \mathrm{~Hz}, 1 \mathrm{H}, \mathrm{CH}_{\mathrm{Ar}}\right), 7.84-7.86(\mathrm{~m}, 3 \mathrm{H}$, $\left.3 \times \mathrm{CH}_{\mathrm{Ar}}\right)$.

${ }^{13} \mathrm{C} \mathrm{NMR}\left(150 \mathrm{MHz}, \mathrm{CDCl}_{3}\right): \delta=21.7\left(\mathrm{CH}_{3}\right), 61.2(\mathrm{CHPh}), 85.3\left(\mathrm{CHNO}_{2}\right)$, $116.3\left(\mathrm{~d},{ }^{2} J_{\mathrm{C}, \mathrm{F}}=24.3 \mathrm{~Hz}, \mathrm{CH}_{\mathrm{Ar}}\right), 121.6\left(\mathrm{~d},{ }^{2} J_{\mathrm{C}, \mathrm{F}}=22.5 \mathrm{~Hz}, \mathrm{CH}_{\mathrm{Ar}}\right), 123.6(\mathrm{~d}$, $\left.{ }^{4} J_{\mathrm{C}, \mathrm{F}}=3.8 \mathrm{~Hz}, \mathrm{C}_{\mathrm{Ar}}\right), 126.3\left(2 \times \mathrm{CH}_{\mathrm{Ar}}\right), 129.1\left(\mathrm{CH}_{\mathrm{Ar}}\right), 129.3\left(2 \times \mathrm{CH}_{\mathrm{Ar}}\right)$, $129.4\left(2 \times \mathrm{CH}_{\mathrm{Ar}}\right), 129.6\left(2 \times \mathrm{CH}_{\mathrm{Ar}}\right), 131.2\left(\mathrm{~d},{ }^{3} \mathrm{~J}_{\mathrm{C}, \mathrm{F}}=7.8 \mathrm{~Hz}, \mathrm{C}_{\mathrm{Ar}}\right), 132.9(\mathrm{~d}$, $\left.{ }^{3} J_{\mathrm{C}, \mathrm{F}}=8.3 \mathrm{~Hz}, \mathrm{CH}_{\mathrm{Ar}}\right), 134.8\left(\mathrm{C}_{\mathrm{Ar}}\right), 134.9\left(\mathrm{C}_{\mathrm{Ar}}\right), 145.7\left(\mathrm{C}_{\mathrm{Ar}}\right), 160.0\left(\mathrm{~d},{ }^{4} J_{\mathrm{C}, \mathrm{F}}=\right.$ $1.8 \mathrm{~Hz}, \mathrm{C}=0), 164.3\left(\mathrm{~d},{ }^{1} \mathrm{~J}_{\mathrm{C}, \mathrm{F}}=254.3 \mathrm{~Hz}, \mathrm{C}_{\mathrm{Ar}} \mathrm{F}\right)$.

${ }^{19} \mathrm{~F}$ NMR (565 MHz, $\mathrm{CDCl}_{3}$ ): $\delta=-105.8\left(\mathrm{ddd},{ }^{3} J_{\mathrm{H}, \mathrm{F}}=8.0,8.0 \mathrm{~Hz},{ }^{4} \mathrm{~J}_{\mathrm{H}, \mathrm{F}}=\right.$ $\left.5.0 \mathrm{~Hz}, \mathrm{C}_{\mathrm{Ar}} \mathrm{F}\right)$.

MS (EI, $70 \mathrm{eV}): m / z(\%)=441(3)[\mathrm{M}+\mathrm{H}]^{+}, 394(14), 376$ (11), 330 (100), 239 (8), 183 (20), 155 (18), 148 (15), 91 (5), 77 (3).

HRMS: $m / z[\mathrm{M}+\mathrm{H}]^{+}$calcd for $\mathrm{C}_{22} \mathrm{H}_{18} \mathrm{~N}_{2} \mathrm{O}_{5} \mathrm{SF}^{+}$: 441.0915; found: 441.0916 .

\section{(3R,4S)-4-Nitro-2-tosyl-3-(1-tosyl-1H-indol-3-yl)-3,4-dihydroiso- quinolin-1(2H)-one (111)}

Synthesized by dissolving 2-(nitromethyl)benzaldehyde (6a; $51 \mathrm{mg}$, $0.31 \mathrm{mmol}$ ), $N$-tosylimine 91 (149 mg, $0.33 \mathrm{mmol}$ ) and $\mathrm{Et}_{3} \mathrm{~N}(32 \mathrm{mg}$, $0.32 \mathrm{mmol}$ ) in toluene $(1 \mathrm{~mL})$. The mixture was stirred at $-20^{\circ} \mathrm{C}$ for 2 $\mathrm{d}$. The oxidation step was conducted with PCC (115 mg, $0.53 \mathrm{mmol})$ at r.t. and monitored by TLC. Purification was by flash chromatography on silica gel ( $n$-hexane-EtOAc, 5:1 to 2:1); yield: $84 \mathrm{mg}$ (44\%); colorless solid; $\mathrm{mp} 224{ }^{\circ} \mathrm{C}(i-\mathrm{PrOH})$.

$R_{f}=0.60$ (n-hexane-EtOAc, $\left.1: 1\right)$.
IR (film): 1693, 1596, 1557, 1448, 1399, 1360, 1289, 1244, 1170, $1118,1089,1062,1011,971,840,809,726,702 \mathrm{~cm}^{-1}$.

${ }^{1} \mathrm{H}$ NMR $\left(600 \mathrm{MHz}, \mathrm{CDCl}_{3}\right): \delta=2.30\left(\mathrm{~s}, 3 \mathrm{H}, \mathrm{CH}_{3}\right), 2.43\left(\mathrm{~s}, 3 \mathrm{H}, \mathrm{CH}_{3}\right)$, $5.84\left(\mathrm{~d},{ }^{3} \mathrm{~J}=2.0 \mathrm{~Hz}, 1 \mathrm{H}, \mathrm{CHNO}_{2}\right), 6.98\left(\mathrm{dd},{ }^{3} \mathrm{~J}=1.9 \mathrm{~Hz},{ }^{4} \mathrm{~J}=1.3 \mathrm{~Hz}, 1 \mathrm{H}\right.$, CHAr), $7.15\left(\mathrm{~d}, 3 \mathrm{~J}=8.5 \mathrm{~Hz}, 2 \mathrm{H}, 2 \times \mathrm{CH}_{\mathrm{Ar}}\right), 7.26-7.28\left(\mathrm{~m}, 2 \mathrm{H}, 2 \times \mathrm{CH}_{\mathrm{Ar}}\right)$, $7.33\left(\mathrm{~d},{ }^{3} \mathrm{~J}=8.2 \mathrm{~Hz}, 2 \mathrm{H}, 2 \times \mathrm{CH}_{\mathrm{Ar}}\right), 7.35-7.38\left(\mathrm{~m}, 2 \mathrm{H}, 2 \times \mathrm{CH}_{\mathrm{Ar}}\right), 7.56-$ $7.60\left(\mathrm{~m}, 3 \mathrm{H}, 3 \times \mathrm{CH}_{\mathrm{Ar}}\right), 7.63$ (ddd, ${ }^{3} J=7.6,7.6 \mathrm{~Hz},{ }^{4} \mathrm{~J}=1.1 \mathrm{~Hz}, 1 \mathrm{H}$, $\left.\mathrm{CH}_{\mathrm{Ar}}\right), 7.69-7.71\left(\mathrm{~m}, 1 \mathrm{H}, \mathrm{CH}_{\mathrm{Ar}}\right), 7.93-7.96\left(\mathrm{~m}, 3 \mathrm{H}, 3 \times \mathrm{CH}_{\mathrm{Ar}}\right), 8.24(\mathrm{dd}$, $\left.{ }^{3} J=7.8 \mathrm{~Hz},{ }^{4} J=1.1 \mathrm{~Hz}, 1 \mathrm{H}, \mathrm{CH}_{\mathrm{Ar}}\right)$.

${ }^{13} \mathrm{C}$ NMR (150 MHz, $\left.\mathrm{CDCl}_{3}\right): \delta=21.6\left(\mathrm{CH}_{3}\right), 21.8\left(\mathrm{CH}_{3}\right), 55.1(\mathrm{CHAr})$, $83.5\left(\mathrm{CHNO}_{2}\right), 114.3\left(\mathrm{CH}_{\mathrm{Ar}}\right), 117.9\left(\mathrm{C}_{\mathrm{Ar}}\right), 118.5\left(\mathrm{CH}_{\mathrm{Ar}}\right), 124.1\left(\mathrm{CH}_{\mathrm{Ar}}\right)$, $125.8\left(\mathrm{CH}_{\mathrm{Ar}}\right), 126.0\left(\mathrm{CH}_{\mathrm{Ar}}\right), 126.9\left(2 \times \mathrm{CH}_{\mathrm{Ar}}\right), 127.1\left(\mathrm{C}_{\mathrm{Ar}}\right), 127.5\left(\mathrm{C}_{\mathrm{Ar}}\right)$, $128.1\left(\mathrm{C}_{\mathrm{Ar}}\right), 129.4\left(4 \times \mathrm{CH}_{\mathrm{Ar}}\right), 129.7\left(\mathrm{CH}_{\mathrm{Ar}}\right), 129.9\left(2 \times \mathrm{CH}_{\mathrm{Ar}}\right), 130.7$ $\left(\mathrm{CH}_{\mathrm{Ar}}\right), 131.8\left(\mathrm{CH}_{\mathrm{Ar}}\right), 134.2\left(\mathrm{C}_{\mathrm{Ar}}\right), 134.3\left(\mathrm{CH}_{\mathrm{Ar}}\right), 135.1\left(\mathrm{C}_{\mathrm{Ar}}\right), 135.3\left(\mathrm{C}_{\mathrm{Ar}}\right)$, $145.3\left(\mathrm{C}_{\mathrm{Ar}}\right), 145.6\left(\mathrm{C}_{\mathrm{Ar}}\right), 160.6(\mathrm{C}=0)$.

MS (EI, $70 \mathrm{eV}): m / z(\%)=615(1)\left[\mathrm{M}^{+}\right], 569(6), 414$ (6), 299 (4), 232 (16), 155 (51), 91 (100), 89 (25), 77 (10), 65 (67), 63 (17).

HRMS: $m / z[\mathrm{M}+\mathrm{H}]^{+}$calcd for $\mathrm{C}_{31} \mathrm{H}_{26} \mathrm{~N}_{3} \mathrm{O}_{7} \mathrm{~S}_{2}{ }^{+}$: 616.1207; found: 616.1207.

\section{Acknowledgment}

Support from the European Research Council (ERC Advanced Grant 320493 'DOMINOCAT') is gratefully acknowledged.

\section{Supporting Information}

Supporting information for this article is available online at http://dx.doi.org/10.1055/s-0034-1379398.

\section{References}

(1) (a) Shamma, M. Isoquinoline Alkaloids, Chemistry and Pharmacology; Academic: New York, 1972. (b) Müjde, B.; Özcan, S.; Balci, M. Phytochemistry Lett. 2011, 4, 407.

(2) For the isolation of thalflavine, see: (a) Umarov, K. S.; Ismailov, Z. F.; Yunusov, S. Y. Chem. Nat. Compd. 1970, 6, 452. (b) Popović, M.; Djurković, R.; Gašić, O.; Pal, B.; Dutschewska, H.; Kuzmanov, B. Biochem. Syst. Ecol. 1992, 20, 255. For the revision of the proposed structure, see: (c) Aly, Y.; Galal, A.; Wong, L. K.; Fu, E. W.; Lin, F.-T.; Duah, F. K.; Schiff, P. L. Jr. Phytochemistry 1989, 28, 1967.

(3) For the isolation of pancratistatin, see: (a) Pettit, G. R.; Gaddamidi, V.; Cragg, G. M.; Herald, D. L.; Sagawa, Y. J. Chem. Soc., Chem. Commun. 1984, 1693. (b) Pettit, G. R.; Gaddamidi, V.; Cragg, G. M. J. Nat. Prod. 1984, 47, 1018. (c) Pettit, G. R.; Gaddamidi, V.; Herald, D. L.; Singh, S. B.; Cragg, G. M.; Schmidt, J. M.; Boettner, F. E.; Williams, M.; Sagawa, Y. J. Nat. Prod. 1986, 49, 995. For selected total syntheses, see: (d) Danishefsky, S.; Lee, J. Y. J. Am. Chem. Soc. 1989, 111, 4829. (e) Tian, X.; Hudlicky, T.; Königsberger, K. J. Am. Chem. Soc. 1995, 117, 3643. (f) Rigby, J. H.; Maharoof, U. S. M.; Mateo, M. E. J. Am. Chem. Soc. 2000, 122, 6624. (g) Ko, H. J.; Kim, E.; Park, J. E.; Kim, D.; Kim, S. J. Org. Chem. 2004, 69, 112. (h) Dam, J. H.; Madsen, R. Eur. J. Org. Chem. 2009, 4666. (i) Jung, Y.-G.; Kang, H.-U.; Cho, H.-K.; Cho, C.-G. Org. Lett. 2011, 13, 5890. For selected references on the biological activity, see: (j) Pettit, G. R.; Pettit, G. R. III; Backhaus, R. A.; Boyd, M. R.; Meerow, A. W. J. Nat. Prod. 1993, 56, 1682. (k) Gabrielsen, B.; Montath, T. P.; Huggins, J. W.; Kefauver, D. F.; 
Pettit, G. R.; Groszek, G.; Hollingshead, M.; Kirsi, J. J.; Shannon, W. M.; Schubert, E. M.; Dare, J.; Ugarkar, B.; Ussery, M. A.; Phelan, M. J. J. Nat. Prod. 1992, 55, 1569.

(4) For the isolation of plicamine, see: (a) Ünver, N.; Gözler, T.; Walch, N.; Gözler, B.; Hesse, M. Phytochemistry 1999, 50, 1255. For reported total syntheses, see: (b) Baxendale, I. R.; Ley, S. V.; Piutti, C. Angew. Chem. Int. Ed. 2002, 41, 2194. (c) Baxendale, I. R.; Ley, S. V.; Nessi, M.; Piutti, C. Tetrahedron 2002, 58, 6285.

(5) Leese, M. P.; Jourdan, F. L.; Major, M. R.; Dohle, W.; Thomas, M. P.; Hamel, E.; Ferrandis, E.; Mahon, M. F.; Newman, S. P.; Purohit, A.; Potter, B. V. L. ChemMedChem 2014, 9, 798.

(6) Zhou, D.; Gross, J. L.; Adedoyin, A. B.; Aschmies, S. B.; Brennan, J.; Bowlby, M.; Di, L.; Kubek, K.; Platt, B. J.; Wang, Z.; Zhang, G.; Brandon, N.; Comery, T. A.; Robichaud, A. J. J. Med. Chem. 2012, $55,2452$.

(7) For selected, recent examples of the preparation of dihydroisoquinolinones, see: (a) Tietze, L. F.; Burkhardt, O. Liebigs Ann. 1995, 1153. (b) Miura, T.; Morimoto, M.; Yamauchi, M.; Murakami, M. J. Org. Chem. 2010, 75, 5359. (c) Srivastava, A. K.; Song, H.; Park, S. B. Synthesis 2011, 2215. (d) Chen, P.-Y.; Chen, H.-M.; Chiang, M. Y.; Wang, Y.-F.; Li, S.-R.; Wang, T.-P.; Wang, E.C. Tetrahedron 2012, 68, 3030. (e) Chen, W.; Cui, J.; Zhu, Y.; Hu, X.; Mo, W. J. Org. Chem. 2012, 77, 1585. (f) Li, B.; Ma, J.; Wang, N.; Feng, H.; Xu, S.; Wang, B. Org. Lett. 2012, 14, 736. (g) Tang, Q.; Xia, D.; Jin, X.; Zhang, Q.; Sun, X.-Q.; Wang, C. J. Am. Chem. Soc. 2013, 135, 4628. (h) Nieto-García, O.; Alonso, R. J. Org. Chem. 2013, 78, 2564. (i) Petrone, D. A.; Yoon, H.; Weinstabl, H.; Lautens, M. Angew. Chem. Int. Ed. 2014, 53, 7908.

(8) Enders, D.; Braig, V.; Boudou, M.; Raabe, G. Synthesis 2004, 2980.

(9) For selected reviews on organocatalytic domino/cascade reactions, see: (a) Enders, D.; Grondal, C.; Hüttl, M. R. M. Angew. Chem. Int. Ed. 2007, 46, 1570. (b) Walji, A. M.; MacMillan, D. W. C. Synlett 2007, 1477. (c) Grondal, C.; Jeanty, M.; Enders, D. Nat. Chem. 2010, 2, 167. (d) Albrecht, Ł.; Jiang, H.; Jørgensen, K. A. Angew. Chem. Int. Ed. 2011, 50, 8492. (e) Lu, L.-Q.; Chen, J.-R.; Xiao, W.-J. Acc. Chem. Res. 2012, 45, 1278. (f) Pellissier, H. Adv. Synth. Catal. 2012, 354, 237. (g) Goudedranche, S.; Raimondi, W.; Bugaut, X.; Constantieux, T.; Bonne, D.; Rodriguez, J. Synthesis 2013, 45, 1909. (h) Chauhan, P.; Enders, D. Angew. Chem. Int. Ed. 2014, 53, 1485. (i) Volla, C. M. R.; Atodiresei, I.; Rueping, M. Chem. Rev. 2014, 114, 2390.

(10) Enders, D.; Hahn, R.; Atodiresei, I. Adv. Synth. Catal. 2013, 355 , 1126.

(11) For selected reviews and examples of hydrogen-bonding catalysis, see: (a) Schreiner, P. R. Chem. Soc. Rev. 2003, 32, 289. (b) Taylor, M. S.; Jacobsen, E. N. Angew. Chem. Int. Ed. 2006, 45, 1520. (c) Connon, S. J. Chem. Eur. J. 2006, 12, 5418. (d) Doyle, A. G.; Jacobsen, E. N. Chem. Rev. 2007, 107, 5713. (e) Zhang, Z.; Schreiner, P. R. Chem. Soc. Rev. 2009, 38, 1187. (f) EtzenbachEffers, K.; Berkessel, A. Top. Curr. Chem. 2010, 291, 1. (g) Aleman, J.; Parra, A.; Jiang, H.; Jørgensen, K. A. Chem. Eur. J. 2011, 17, 6890. (h) Storer, R. I.; Aciro, C.; Jones, L. H. Chem. Soc. Rev. 2011, 40, 2330. (i) Enders, D.; Urbanietz, G.; Hahn, R.; Raabe, G. Synthesis 2012, 44, 773. (j) Albrecht, Ł.; Dickmeiss, G.; Acosta, F. C.; Rodriguez-Escrich, C.; Davis, R. L.; Jørgensen, K. A. J. Am. Chem. Soc. 2012, 134, 2543. (k) Enders, D.; Urbanietz, G.; CassensSasse, E.; Kees, S.; Raabe, G. Adv. Synth. Catal. 2012, 354, 1481. (l) Loh, C. C. J.; Hack, D.; Enders, D. Chem. Commun. 2013, 49,
10230. (m) Loh, C. C. J.; Atodiresei, I.; Enders, D. Chem. Eur. J. 2013, 19, 10822. (n) Liu, Y.; Wang, Y.; Song, H.; Zhou, Z.; Tang, C. Adv. Synth. Catal. 2013, 355, 2544. (o) Gosh, A. K.; Zhou, B. Tetrahedron Lett. 2013, 54, 3500. (p) Sun, W.; Hong, L.; Zhu, G.; Wang, Z.; Wie, X.; Ni, J.; Wang, R. Org. Lett. 2014, 16, 544. (q) Zhou, E.; Liu, B.; Dong, C. Tetrahedron: Asymmetry 2014, 25, 181. (r) Urbanietz, G.; Atodiresei, I.; Enders, D. Synthesis 2014, 46, 1261. (s) Han, X.; Dong, C.; Zhou, H.-B. Adv. Synth. Catal. 2014, 356, 1275. (t) Chauhan, P.; Mahajan, S.; Loh, C. C. J.; Raabe, G.; Enders, D. Org. Lett. 2014, 16, 2954. (u) Hahn, R.; Raabe, G.; Enders, D. Org. Lett. 2014, 16, 3636.

(12) For a review on aza-Henry reactions, see: (a) Noble, A.; Anderson, J. C. Chem. Rev. 2013, 113, 2887. For selected, catalytic aza-Henry reactions using metal complexes, see: (b) Knudsen, K. R.; Risgaard, T.; Nishiwaki, N.; Gothelf, K. V.; Jørgensen, K. A. J. Am. Chem. Soc. 2001, 123, 5843. (c) Lee, A.; Kim, W.; Lee, J.; Hyeon, T.; Kim, B. M. Tetrahedron: Asymmetry 2004, 15, 2595. (d) Palomo, C.; Oibarbide, M.; Halder, R.; Laso, A.; López, R. Angew. Chem. Int. Ed. 2006, 45, 117. (e) Trost, B. M.; Lupton, D. W. Org. Lett. 2007, 9, 2023. (f) Handa, S.; Gnanadesikan, V.; Matsunaga, S.; Shibasaki, M. J. Am. Chem. Soc. 2007, 129, 4900. (g) Chen, Z.; Morimoto, H.; Matsunaga, S.; Shibasaki, M. J. Am. Chem. Soc. 2008, 130, 2170. (h) Handa, S.; Gnanadesikan, V.; Matsunaga, S.; Shibasaki, M. J. Am. Chem. Soc. 2010, 132, 4925. (i) Blay, G.; Escamilla, A.; Hernández-Olmos, V.; Pedro, J. R.; Sanz-Marco, A. Chirality 2012, 24, 441. (j) Arai, T.; Matsumura, E. Synlett 2014, 25, 1776. (k) Choudhary, M. K.; Das, A.; Kureshy, R. I.; Kumar, M.; Khan, N. H.; Abdi, S. H. R.; Bajaj, H. C. Catal. Sci. Technol. 2014, 4, 548. For selected, organocatalytic aza-Henry reactions, see: (l) Okino, T.; Nakamura, S.; Furukawa, T.; Takemoto, Y. Org. Lett. 2004, 6, 625. (m) Fini, F.; Sgarzani, V.; Pettersen, D.; Herrera, R. P.; Bernardi, L.; Ricci, A. Angew. Chem. Int. Ed. 2005, 44, 7975. (n) Rueping, M.; Antonchick, A. P. Org. Lett. 2008, 10, 1731. (o) Wilt, J.; Pink, M.; Johnston, J. N. Chem. Commun. 2008, 4177. (p) Davis, T. A.; Johnston, J. N. Chem. Sci. 2011, 2, 1076. (q) Wei, Y.; He, W.; Liu, Y.; Liu, P.; Zhang, S. Org. Lett. 2012, 14, 704. (r) Parra, A.; Alfaro, R.; Marzo, L.; MorenoCarrasco, A.; Ruano, J. L. G.; Alemán, J. Chem. Commun. 2012, 48, 9759. (s) Cao, D.; Chai, Z.; Zhang, J.; Ye, Z.; Xiao, H.; Wang, H.; Chen, J.; Wu, X.; Zhao, G. Chem. Commun. 2013, 49, 5972. (t) Núñez, M. G.; Farley, A. J. M.; Dixon, D. J. J. Am. Chem. Soc. 2013, 135, 16348.

(13) For the concept of organocatalyzed aza-Henry/hemiaminalization domino reactions, see: (a) Urushima, T.; Sakamoto, D.; Ishikawa, H.; Hayashi, Y. Org. Lett. 2010, 12, 4588. (b) Imashiro, R.; Uehara, H.; Barbas, C. F. III Org. Lett. 2010, 12, 5250. (c) Rai, A.; Singh, A. K.; Singh, S.; Yadav, L. D. S. Synlett 2011, 335. (d) Rai, A.; Singh, A. K.; Singh, P.; Yadav, L. D. S. Tetrahedron Lett. 2011, 52, 1354. (e) Chawla, R.; Rai, A.; Singh, A. K.; Yadav, L. D. S. Tetrahedron Lett. 2012, 53, 5323. (f) Lin, H.; Tan, Y.; Liu, W.-J.; Zhang, Z.-C.; Sun, X.-W.; Lin, G.-Q. Chem. Commun. 2013, 49, 4024.

(14) (a) Enders, D.; Urbanietz, G.; Raabe, G. Synthesis 2011, 1905. For a closely related catalyst, see: (b) Flock, A. M.; Krebs, A.; Bolm, C. Synlett 2010, 1219.

(15) CCDC 1027768 (11a) contains the supplementary crystallographic data for this paper. These data can be obtained free of charge from the Cambridge Crystallographic Data Centre via www.ccdc.cam.ac.uk/data_request/cif. 